\title{
Farsighted manipulation and exploitation in networks
}

Citation for published version (APA):

Bayer, P., Herings, P. J-J., \& Peeters, R. (2019). Farsighted manipulation and exploitation in networks. Maastricht University, Graduate School of Business and Economics. GSBE Research Memoranda No. 023 https://doi.org/10.26481/umagsb.2019023

Document status and date:

Published: 29/08/2019

DOI:

10.26481/umagsb.2019023

Document Version:

Publisher's PDF, also known as Version of record

\section{Please check the document version of this publication:}

- A submitted manuscript is the version of the article upon submission and before peer-review. There can be important differences between the submitted version and the official published version of record.

People interested in the research are advised to contact the author for the final version of the publication, or visit the DOI to the publisher's website.

- The final author version and the galley proof are versions of the publication after peer review.

- The final published version features the final layout of the paper including the volume, issue and page numbers.

Link to publication

\footnotetext{
General rights rights.

- You may freely distribute the URL identifying the publication in the public portal. please follow below link for the End User Agreement:

www.umlib.nl/taverne-license

Take down policy

If you believe that this document breaches copyright please contact us at:

repository@maastrichtuniversity.nl

providing details and we will investigate your claim.
}

Copyright and moral rights for the publications made accessible in the public portal are retained by the authors and/or other copyright owners and it is a condition of accessing publications that users recognise and abide by the legal requirements associated with these

- Users may download and print one copy of any publication from the public portal for the purpose of private study or research.

- You may not further distribute the material or use it for any profit-making activity or commercial gain

If the publication is distributed under the terms of Article $25 \mathrm{fa}$ of the Dutch Copyright Act, indicated by the "Taverne" license above, 


\section{Maastricht University}

Péter Bayer,

P. Jean-Jacques Herings,

Ronald Peeters

Farsighted manipulation and exploitation in networks

RM/19/023

\section{GSBE}

Maastricht University School of Business and Economics

Graduate School of Business and Economics

P.O Box 616

NL- 6200 MD Maastricht

The Netherlands 


\title{
Farsighted manipulation and exploitation in networks*
}

\author{
Péter Bayer $^{\dagger} \quad$ P. Jean-Jacques Herings ${ }^{\ddagger} \quad$ Ronald Peeters ${ }^{\S}$
}

August 22, 2019

\begin{abstract}
Economic agents with an increased sophistication sometimes use their advantage to exploit their more naive counterparts. In public goods games played on networks, such an agent will attempt to manipulate as many of his neighbors as possible to produce the public good. We study the exploitation of a myopic population by a single farsighted player in such games. We show the existence and payoff-uniqueness of optimal farsighted strategies in every network structure. In the long run, the farsighted player's effects are only felt locally. A simple dependence-withdrawal strategy reaches the optimal outcome for every network if the starting state is unfavorable, and reaches it for every starting state if the farsighted player is linked to all opponents. We characterize the lower and upper bounds of long-run payoffs the farsighted player can attain in a given network and make comparative statics with respect to adding a new link. The farsighted player always benefits from linking to more opponents (sociability) and is always harmed by his neighbors linking to each other (jealousy).
\end{abstract}

Keywords: Networks, Public goods, Myopic and farsighted players.

JEL classification: C73, D85, H41.

\footnotetext{
${ }^{*}$ This project was partially financed by the Centre for Human Enhancement and Learning of Maastricht University. Péter Bayer thanks Aix-Marseille School of Economics for its hospitality, sponsored by the Graduate School of Business and Economics of Maastricht University. We thank Yann Bramoullé, Péter Csóka, László Kóczy, and Frank Thuijsman for comments on previous versions of this paper.

${ }^{\dagger}$ University of Grenoble-Alpes, GAEL, 1241 Rue des Résidences, 38400 Saint Martin d'Hères, France. E-mail: peter.bayer@univ-grenoble-alpes.fr.

${ }^{\ddagger}$ Department of Economics, Maastricht University, P.O. Box 616, 6200 MD Maastricht, The Netherlands. E-mail: p.herings@maastrichtuniversity.nl.

${ }^{\S}$ Department of Economics, University of Otago, P.O. Box 56, Dunedin 9054, New Zealand. E-mail: ronald.peeters@otago.ac.nz.
} 


\section{Introduction}

In many social and economic settings, agents produce goods for their own consumption with other agents also benefitting from these goods as a form of externality. The typical free-rider problem results from agents trying to benefit from goods produced by others while not producing any themselves. Oftentimes the goods produced by one agent may affect some but not all other agents. This heterogeneity in the interaction structure is typically captured by the local public goods model with the agents' positions being governed by a network.

Economic situations involving local public goods often pit players with different levels of sophistication, ability, or time horizon against one another. In such cases, players on a higher level may find it profitable to engage in strategic free riding; rather than producing their best-response amounts of the good, they produce less, sacrificing short-run consumption gains in order to manipulate their low-level opponents to raise production and thus exploit their disadvantage. Depending on the specifics of the game and the interaction structure, the strategies of manipulation and exploitation may be much more complicated. In this paper, we study how these strategies and the gains from exploitation possibilities depend upon the network. This approach allows us not only to find optimal methods of exploitation and manipulation, but also to identify social environments in which such strategies produce the greatest gains.

The class of local public goods games has an established theoretical literature, starting with Ballester et al. (2006), and a wide applicability with contributions on the distribution of R\&D costs between interlinked firms (König et al. 2019), criminal activity (Calvó-Armengol and Zenou, 2004), peer effects (Blume et al. 2010), and defense/counter-terrorism expenditure between allies (Sandler and Hartley 1995, 2007).

For the situation where agents are homogenous in their reasoning, local public good games have a rich recent literature. Bramoullé and Kranton (2007) shows existence of Nash equilibria and that equilibria with free riders are present in any non-trivial network. The follow-up paper, Bramoullé et al. (2014) provides conditions such that stable Nash equilibria with respect to continuous best-response dynamics exist. Allouch (2015) studies the effects of wealth redistribution. Galeotti and Goyal (2010) and Choi et al. (2019) study players who can invest in both public good provision and building up their connections. Goyal (2012) and Jackson and Zenou (2014) offer comprehensive overviews of additional related models. A strand of literature focuses on the convergence of learning processes. It has been shown that best-response dynamics converge to a single Nash equilibrium (Bervoets and Faure, 2019) and that convergence may be achieved by a learning process with very little requirements on player sophistication (Bervoets et al. 2016). Bayer et al. (2019) shows such convergence for a class of learning processes. Parise and Özdaglar (2019) extends Bramoullé et al. (2014)'s results by the use of variational inequalities.

In the spirit of Ellison (1997) we include a single player of increased sophistication into a population of naive agents. In games of strategic complements such as coordination games, the addition of the 
more sophisticated player improves efficiency as coordination shifts towards a better Nash equilibrium by performing strategic teaching (Camerer et al. 2002). In contrast, our game, a game of local strategic substitutes, induces an exploitative behavior from the sophisticated player with no clear gains or losses in efficiency. To be precise, we consider the presence of one farsighted player in a population of myopic players. The farsighted player's interest lies in the exploitation of the myopic players by maximizing his neighbors' production of the local public good, while minimizing his own efforts. Many recent papers study farsightedness in general environments by the use of the farsighted stable set (Ray and Vohra, 2015) and its refinements (Dutta and Vohra, 2017; Bloch and van den Nouweland, 2019), as well as in the context of coalition formation (Mauleon and Vannetelbosch, 2004), network formation (Dutta et al. 2005; Page et al., 2005, Herings et al., 2009), and matching (Klaus et al., 2011). This approach can be extended to the myopic-farsighted stable set to capture the interaction between these two types of players (Herings et al., 2017; Luo et al., 2018). Our paper also studies such interactions.

To illustrate the manipulation and exploitation strategies of a farsighted player, consider a game played by four players, $0,1,2$, and 3 . They inhabit a network in which all players are linked to all others except for players 1 and 3, called the "kite network", see Figure1. Each player may exert costly efforts into creating a perfectly substitutable good which is then freely accessible for all of their neighbors in the network. The players have no efficient ways to exclude their neighbors from sharing the benefits of their efforts, nor does the neighbors' consumption harm the producer in any way, hence we think of these goods as local public goods. We impose the normalization that marginal costs of production equal marginal benefits of consumption when players consume 1 unit of the good. Thus, every player's best response is to set an effort that guarantees a consumption of 1 , or set an effort of 0 , if neighboring efforts exceed 1. We consider two situations. In the first situation, player 2 exerts an effort of 1 and the other three players exert 0 effort. In the second situation, players 1 and 3 exert an effort of 1 and the others exert 0 effort, see Figure 1 .
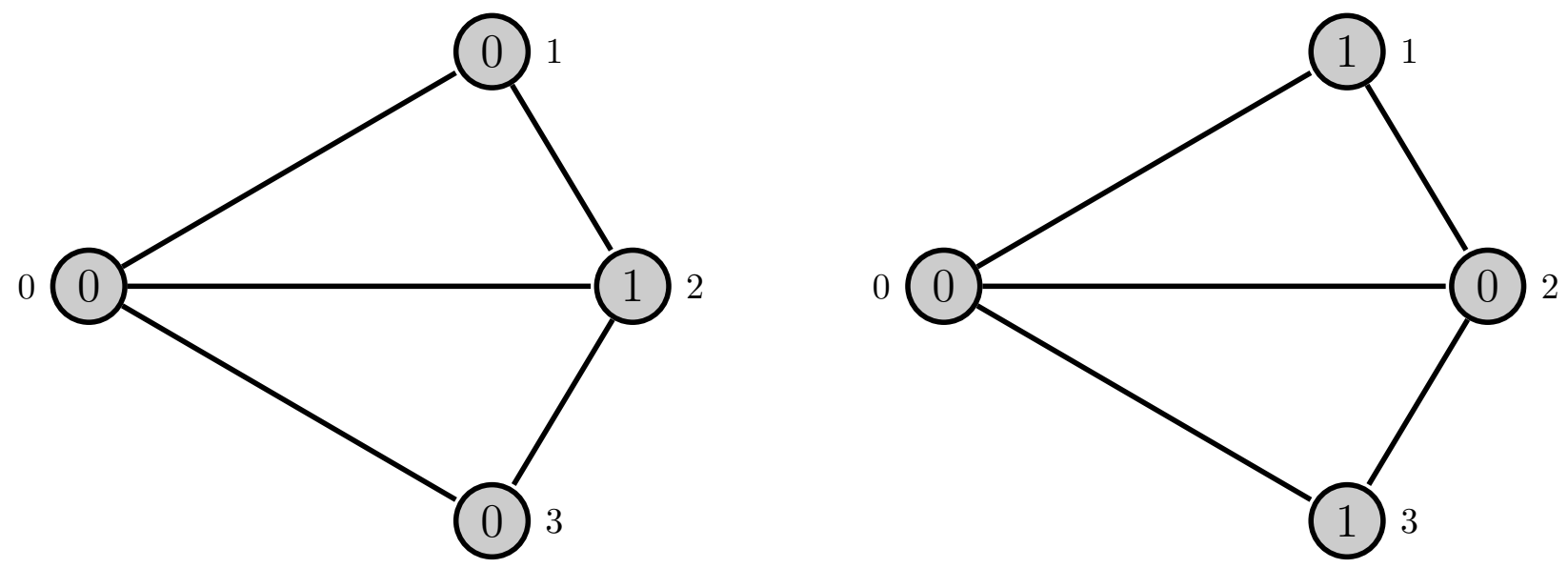

Figure 1: The kite network.

In both situations, every player is at a static best response as marginal costs either equal marginal benefits for producers, while the free riders receive at least their target level of the local public good 
from neighboring producers. Therefore, despite the obvious inequalities between players, myopic players would not deviate in either situation. Notice, however, that for player 0 , the second situation is better than the first, as he gets twice the amount of production from his neighbors without incurring extra costs.

Assume that players 1,2, and 3 are myopic, while player 0 is farsighted and is fully informed of the opponents' myopia. Suppose that, starting from the first situation, player 0 increases his efforts to 1 . Then once player 2 , being the only other producer, is able to change his own effort level, he will cease production completely. Now suppose that player 0 ceases as well, reaching a state with no effort from any player. Then, depending on the timing of the others' reactions, two outcomes may be reached. If player 2 reacts to the new situation first, we return to the first situation, upon which player 0 may repeat the process. If player 1 (player 3 ) reacts first, he will raise his efforts to 1 , upon which only player 3 (player 1) can deviate profitably by doing the same, reaching the second situation. Thus, player 0 has a way to secure a long-run consumption level of 2 , the maximum long-run consumption he can freely receive in this network. The strategy giving the improvement is simple, first produce for his neighborhood, creating dependence, followed by a withdrawal.

It is clear that a farsighted player's ability to free ride on as many of his neighbors as possible depends on his position in the network; given the first situation, adding the link $\{1,3\}$ would mean that player 0 cannot improve upon a consumption of 1 , while removing the link $\{0,2\}$ would mean that his consumption needs will have to be covered through his own efforts as both of his neighbors are kept at 0 effort level; they are "blocked" by player 2's efforts, see Figure 2 .
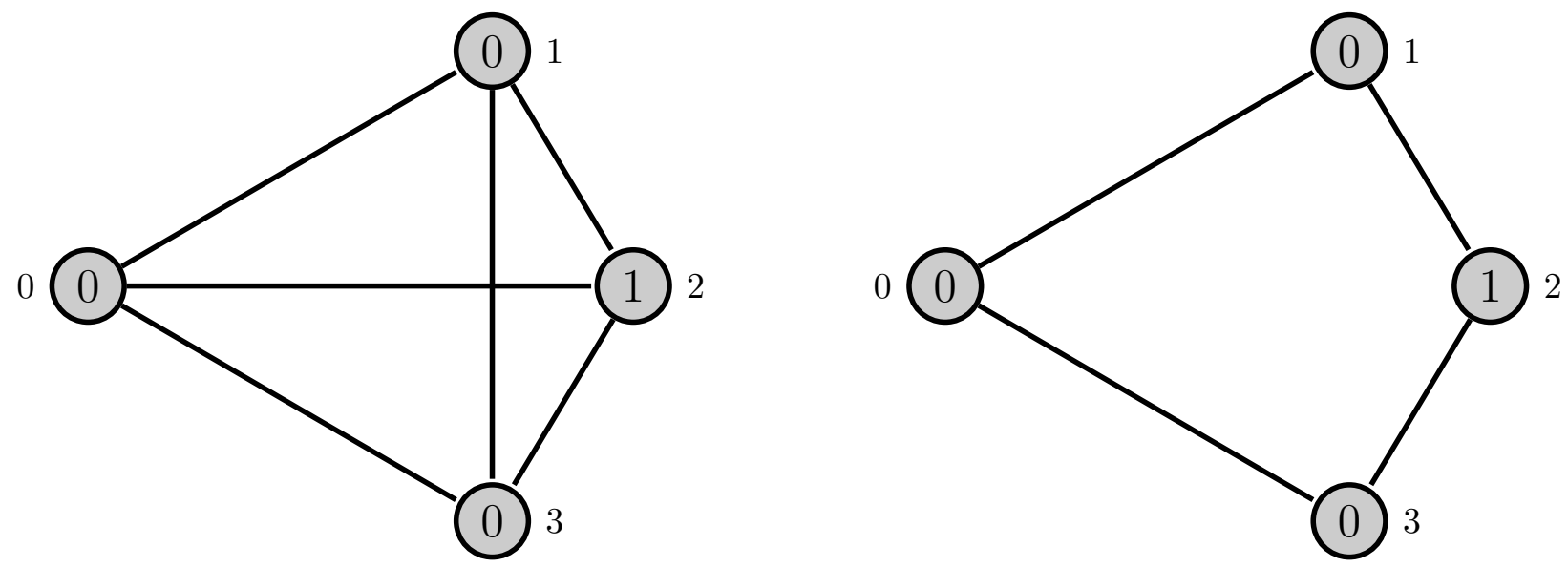

Figure 2: Two variants of the kite network with a link added and a link removed. Both harm player 0's prospects for exploitation.

We study exploitation strategies of the above kind in general spatial structures where one farsighted player faces a myopic population. Our setting is a dynamic one, taking place in discrete time. In every period a randomly selected player has a chance to revise his exerted effort. Once he does, period payoffs are awarded, and a new random player receives a revision opportunity. Myopic players maximize their instantaneous payoffs, while the farsighted player maximizes the discounted sum of instantaneous 
payoffs.

Our contributions are as follows: Using classic results of Markov Decision Processes we show existence of optimal exploitation strategies by the farsighted player for every network structure. In addition, we also show existence of equilibrium in outcomes; under equilibrium play there exist a set of rest points of the dynamic process. This set does not necessarily coincide with the set of Nash equilibria in the static game since (1) some Nash equilibria of the static game can be improved upon by the farsighted player as seen above and (2) effort profiles in which myopic players are playing their current best responses may be profitable for the farsighted player even if he is not at his best response. Thus the set of absorbing effort profiles is neither a subset nor a superset of the set of Nash equilibria of the static game.

Next, we find that the farsighted player's effect on the population is only felt locally in the long run; every myopic player who does not share a neighbor with the farsighted player, or isn't a neighbor himself will, in time, settle for a constant effort output. The reason behind this is that autonomous islands of myopic producers always emerge outside the neighborhood of the farsighted player surrounded by a layer of free riders sustained entirely by the island. These free riders dampen any effects arriving from the outside thus negating the farsighted player's influence. Since a farsighted player's long-run effects are local, our results are robust for multiple farsighted players, provided that they appear sparsely enough in the network.

In the kite network, the farsighted player's optimal outcome is to exploit the myopic neighbors belonging to the maximum independent set of his neighborhood and this outcome can be reached by a simple dependence-withdrawal strategy. We confirm the first fact for every network, thus characterizing an upper bound of the farsighted player's prospects for exploitation. However, for general network structures, exploiting a maximum independent set of the neighborhood is not always attainable for some starting states, e.g., the right side of Figure2, We define the lower bound of farsighted player's prospects as the minimum long-run payoff they can guarantee from any starting state in the given network and characterize it as the maximum unblocked independent set of the farsighted player's neighborhood. We show that the value of the lower bound is always reachable using a dependence-withdrawal strategy. Thus, a very simple strategy does no worse than the optimal strategy in unfavorable starting states.

It is natural to ask how the farsighted player's prospects are changed trough changes in the network. We answer this by considering the comparative statics of a new link to the upper and lower bounds. We find that adding a link to connect the farsighted player to an additional neighbor always weakly increases both the upper and lower bounds, while adding link between any two of his neighbors always weakly decreases them. Thus, the farsighted player has a preference to have a direct link with as many opponents as possible and a preference for his neighbors to be unlinked. We thus found an interesting justification for two human emotions which drive the formation of social connections; sociability, the drive to form as many links as possible, and jealousy, the inclination to be in exclusive relationships, both help farsighted agents to maximize their exploitative potential. 
The paper proceeds as follows. Section 2 introduces local public goods games, introduces the dynamic and stochastic components of the model, shows the existence of equilibrium strategies, and demonstrates the uniqueness of the farsighted player's equilibrium payoffs. Section 3 narrows down the effort profiles that can be recurrent and shows the existence of absorbing effort profiles. The main result of Section 4 establishes that the in the long run the farsighted player has only local impact. Section 5 presents the lower and upper bounds on the payoffs of the farsighted player and contains our comparative statics results. Section 6 concludes.

\section{The model}

We first consider the static local public goods game $\Gamma=\left(I_{0}, X, \pi\right)$, which is determined by the primitives $\left(n, G,\left(f_{i}\right)_{i \in I_{0}},\left(\gamma_{i}\right)_{i \in I_{0}}, d\right)$. We then go on to extend this model to a dynamic game $\Gamma^{\prime}=\left(I_{0}, X, \Omega, q, \delta\right)$ in discrete time, which involves additional primitives $(q, \delta)$.

There are $n \geq 1$ myopic players, which we collect in the set $I=\{1, \ldots, n\}$. There is also a farsighted player, denoted by player 0 . Let the set $I \cup\{0\}$ be denoted by $I_{0}$. A network structure, characterized by the binary, symmetric matrix $G$, represents the local interactions between the players. For $i, j \in I_{0}$, $g_{i j}=1$ means that players $i$ and $j$ are connected, while $g_{i j}=0$ means that they are not. As a notational convention, we assume $g_{i i}=1$ for all $i \in I_{0}$. For a player $i \in I_{0}$, the set $N_{i}=\left\{j \in I_{0} \backslash\{i\}: g_{i j}=1\right\}$ is called $i$ 's neighborhood. For $J \subseteq I_{0}$, let $N_{J}=\left\{i \in I_{0} \backslash J: g_{i j}=1\right.$ for some $\left.j \in J\right\}$ denote the neighborhood of a set of players $J$. Finally, for $i \in I_{0}$, let $\bar{N}_{i}=N_{i} \cup\{i\}$ and, for $J \subseteq I_{0}$, let $\bar{N}_{J}=N_{J} \cup J$.

Let $d$ be a positive integer and $Z=\{0,1 / d, 2 / d, \ldots\}$. The set of possible effort levels of a player $i \in I_{0}$ is given by $X_{i}=Z$. For $J \subseteq I_{0}$, we define $X_{J}=\prod_{j \in J} X_{j}$. We also use the notation $X$ for the set $X_{I_{0}}$ and, for $i \in I_{0}, X_{-i}$ for the set $X_{I_{0} \backslash\{i\}}$. For an effort profile $x \in X$ and a set of players $J \subseteq I_{0}$, we denote by $x(J)=\sum_{i \in J} x_{i}$ the sum of efforts by the players in $J$. For $i \in I_{0}$, the value $x\left(\bar{N}_{i}\right)$ is called player $i$ 's consumption at profile $x$ and is denoted by $c_{i}(x)$ (also called a neighborhood vector in Allouch and King (2019)).

The players are engaged in a local public goods game $\Gamma=\left(I_{0}, X, \pi\right)$ with payoff functions $\pi_{i}: X \rightarrow \mathbb{R}$ that are given by

$$
\pi_{i}(x)=f_{i}\left(c_{i}(x)\right)-\gamma_{i} x_{i}
$$

where $\gamma_{i} \in \mathbb{R}_{+}$and the functions $f_{i}: Z \rightarrow \mathbb{R}_{+}$satisfy the following properties:

1. $f_{i}(0)=0$.

2. $f_{i}$ is monotonically increasing.

3. $f_{i}\left(\tilde{c}_{i}\right)-f_{i}\left(\tilde{c}_{i}-1 / d\right) \geq f_{i}\left(\tilde{c}_{i}+1 / d\right)-f_{i}\left(\tilde{c}_{i}\right)$ for all $\tilde{c}_{i} \in Z \backslash\{0\}$.

4. $f_{i}(1)-f_{i}(1-1 / d)>\gamma_{i} / d$ and $f_{i}(1+1 / d)-f_{i}(1)<\gamma_{i} / d$. 
For $i \in I_{0}$ and $x \in X$, the value $f_{i}\left(c_{i}(x)\right)$ is called the benefits enjoyed by player $i$ from his consumption $c_{i}(x)$ in profile $x$. The function $f_{i}$ is called the benefit function of player $i$, while the parameter $\gamma_{i}$ is the cost of one unit of effort for player $i$. Let $\pi(x)$ denote the vector of payoffs $\left(\pi_{0}(x), \ldots, \pi_{n}(x)\right)$ in effort profile $x$.

The four properties of the benefit function respectively mean (1) the benefits of consuming zero equal zero, (2) monotonicity, (3) concavity, and (4) the existence of an optimal effort level, above which the marginal cost of effort is larger than the marginal benefit of consumption. These properties are standard in the discussion of local public good games. The optimal effort levels are the same for all players and, by (4), their value is normalized to one.

For the game where efforts are chosen from $\mathbb{R}_{+}$, it is possible to give a constructive proof for the existence of Nash equilibria, see Goyal (2012). The same construction can be used to show the existence of Nash equilibria in the game $\Gamma$, where efforts are chosen from the discrete set $Z$. Take any player $j^{0} \in I_{0}$ and define $x_{j^{0}}^{*}=1$. For every $i \in N_{j^{0}}$, define $x_{i}^{*}=0$. If $\bar{N}_{j^{0}}=I_{0}$, then it is easy to verify that $x^{*} \in X$ is a Nash equilibrium and we are done. Otherwise, take any player $j^{1} \in I_{0} \backslash \bar{N}_{j^{0}}$ and define $x_{j^{1}}^{*}=1$. For every $i \in N_{j^{1}} \backslash \bar{N}_{j^{0}}$, define $x_{i}^{*}=0$. If $\bar{N}_{j^{0}} \cup \bar{N}_{j^{1}}=I_{0}$, then it is easy to verify that $x^{*} \in X$ is a Nash equilibrium. Otherwise, take any player $j^{2} \in I_{0} \backslash\left(\bar{N}_{j^{0}} \cup \bar{N}_{j^{1}}\right)$ and define $x_{j^{2}}^{*}=1$. Repeating this argument finitely many times results in a Nash equilibrium $x^{*}$. We have therefore shown the following result.

Proposition 2.1. The game $\Gamma$ has a Nash equilibrium.

We are interested in the dynamic behavior of a farsighted player acting against a set of myopic players in the dynamic extension of $\Gamma$, denoted by $\Gamma^{\prime}$. The dynamics take place in discrete time, indexed by $t=1,2, \ldots$. The game starts with an arbitrary effort profile, $x^{0} \in X$. Let $q=\left(q_{0}, \ldots, q_{n}\right) \in \mathbb{R}^{n+1}$ be a strictly positive vector with $\sum_{i \in I_{0}} q_{i}=1$. In every period $t=1,2, \ldots$, player $i \in I_{0}$ becomes the active player with probability $q_{i}$ and is denoted by $i^{t}$. The active player $i^{t}$ gets a revision opportunity and updates his effort level, whereas the other players keep their effort level from the previous period, leading to effort profile $x^{t}$ and instantaneous payoffs $\pi\left(x^{t}\right)$ in period $t$.

A state is characterized by the previous period's effort profile and the active player. The set of states is denoted by $\Omega=X \times I_{0}$. For a given effort profile $x \in X$, the effort profile that results if a player $i \in I_{0}$ updates his effort level to $z \in X_{i}$ is denoted by $\left(z, x_{-i}\right)$.

Fix $T \geq 1$. For time periods $1 \leq t \leq T$, states $\omega^{t}=\left(x^{t-1}, i^{t}\right) \in \Omega$, and choices $z^{t} \in X_{i^{t}}$, a sequence $h^{T}=\left(\omega^{1}, z^{1}, \omega^{2}, z^{2}, \ldots, \omega^{T-1}, z^{T-1}, \omega^{T}\right)$ such that for every $1 \leq t \leq T$ it holds that

$$
x_{-i^{t}}^{t}=x_{-i^{t}}^{t-1} \text { and } x_{i^{t}}^{t}=z^{t}
$$

is called a $T$-long history. Let $H^{T}$ denote the set of $T$-long histories and $H=\bigcup_{T \in \mathbb{N}} H^{T}$ denote the set of finite histories. For a player $i \in I_{0}$, an effort profile $x \in X$, and a state $\omega \in \Omega$, let $H_{i}^{T}, H_{x}^{T}$, and $H_{\omega}^{T}$ denote the set of $T$-long histories for which the last active player $i^{T}$ is equal to $i$, the last effort 
profile $x^{T-1}$ is equal to $x$, and the last state $\omega^{T}$ is equal to $\omega$, respectively. Define $H_{i}=\bigcup_{T \in \mathbb{N}} H_{i}^{T}$, $H_{x}=\bigcup_{T \in \mathbb{N}} H_{x}^{T}$, and $H_{\omega}=\bigcup_{T \in \mathbb{N}} H_{\omega}^{T}$.

A strategy of a player $i \in I_{0}$ is a function $\tilde{s}_{i}: H_{i} \rightarrow X_{i}$. For a history $h \in H_{i}$, the value $\tilde{s}_{i}(h)$ is the effort chosen by player $i$ after observing history $h$. Denote the set of strategies of player $i$ by $\tilde{S}_{i}$ and let $\tilde{S}=\prod_{i \in I} \tilde{S}_{i}$ denote the set of strategy profiles.

The transitions between the states are governed by a distribution $Q: \Omega \times Z \rightarrow \Delta(\Omega)$. The probability that state $\omega^{\prime}$ follows $\omega$ if choice $z$ is made equals $Q\left(\omega^{\prime} \mid \omega, z\right)$. For every $x, x^{\prime} \in X, i, i^{\prime} \in I_{0}$, and $z \in X_{i}$ we have

$$
Q\left(\left(x^{\prime}, i^{\prime}\right) \mid(x, i), z\right)= \begin{cases}q_{i^{\prime}} & \text { if } x^{\prime}=\left(z, x_{-i}\right) \\ 0 & \text { otherwise }\end{cases}
$$

For strategy profile $\tilde{s}$ and a $T$-long history $h \in H^{T}$, let $u_{0}^{t}(h, \tilde{s})$ denote the expected instantaneous payoff that player 0 receives in period $T+t-1$. For example, it holds that $u_{0}^{1}(h, \tilde{s})=\pi_{0}\left(\tilde{s}_{i^{T}}(h), x_{-i^{T}}^{T-1}\right)=\pi_{0}\left(x^{T}\right)$. Player 0's utility is given by the sum of discounted expected instantaneous payoffs, where $\delta \in(0,1)$ is the discount factor:

$$
U_{0}(h, \tilde{s})=\sum_{t=1}^{\infty} \delta^{t-1} u_{0}^{t}(h, \tilde{s}) .
$$

A myopic player $i \in I$ cares only about his instantaneous payoffs, so for $x \in X$ and $h \in H^{T}$, his utility function is defined by

$$
U_{i}(h, \tilde{s})=\pi_{i}\left(\tilde{s}_{i^{T}}(h), x_{-i^{T}}^{T-1}\right) .
$$

We start by analyzing the subgame perfect equilibria of the game $\Gamma^{\prime}$, formally defined as follows.

Definition 2.2 (SPE). A strategy profile $\tilde{s}^{*} \in \tilde{S}$ is a subgame perfect equilibrium (SPE) of $\Gamma^{\prime}$ if for every player $i \in I_{0}$, for every history $h \in H$, and for every strategy $\tilde{s}_{i} \in \tilde{S}_{i}$ it holds that

$$
U_{i}\left(h, \tilde{s}^{*}\right) \geq U_{i}\left(h,\left(\tilde{s}_{i}, \tilde{s}_{-i}^{*}\right)\right) .
$$

Let $\tilde{S}^{*}$ denote the set of SPEs. For $i \in I_{0}$, the strategy $\tilde{b}_{i} \in \tilde{S}_{i}$ such that

$$
\tilde{b}_{i}(h)=\max \left\{0,1-x\left(N_{i}\right)\right\}, \quad x \in X, h \in H_{(x, i)},
$$

is called the best-responder strategy of player $i$. Under the best-responder strategy, player $i$ exerts an amount of effort such that his consumption $c_{i}(x)$ is equal to 1 , except when the neighbors of player $i$ together exert more effort than 1, in which case player $i$ does not contribute at all and enjoys a level of consumption above 1 .

The next proposition states that at every SPE the myopic players use the best-responder strategy.

Proposition 2.3. For every $\tilde{s}^{*} \in \tilde{S}^{*}$, for every $i \in I$, it holds that $\tilde{s}_{i}^{*}=\tilde{b}_{i}$.

Proof. The lemma follows immediately from property (4) of the benefit functions $\left(f_{i}\right)_{i \in I}$. 
Note that the best-responder strategy depends only on the last state in a history. Strategies that satisfy this criterion are called stationary strategies. More formally, for $i \in I_{0}$, a strategy $\tilde{s}_{i} \in \tilde{S}_{i}$ is called stationary if there is a function $s_{i}: X \rightarrow X_{i}$ such that, for every $x \in X$, for every $h \in H_{(x, i)}$, it holds that $\tilde{s}_{i}(h)=s_{i}(x)$. The value $s_{i}(x)$ is player $i$ 's choice at effort profile $x$. The collection of such functions $s_{i}$ is denoted by $S_{i}$. From now on, we represent a stationary strategy of player $i$ by an element of $S_{i}$. The member of $S_{i}$ corresponding to the best-responder strategy $\tilde{b}_{i}$ of player $i$ is denoted by $b_{i}$. The effort profile that results from $x$ after player $i$ uses the best-responder strategy is denoted by $\beta_{i}(x)$. Let $S=\prod_{i \in I_{0}} S_{i}$ denote the set of stationary strategy profiles.

A subgame perfect equilibrium in stationary strategies is defined as follows.

Definition 2.4 (SSPE). A strategy profile $s^{*} \in S$ is a subgame perfect equilibrium in stationary strategies (SSPE) of $\Gamma^{\prime}$ if it is an SPE.

Let the set of SSPEs be denoted by $S^{*}$. It follows from Proposition 2.3 that, for every $s^{*} \in S^{*}$, for every $i \in I$, we have $s_{i}^{*}=b_{i}$. In Proposition 2.5 we show that an SSPE exists in this game.

Proposition 2.5. The game $\Gamma^{\prime}$ has an SSPE.

Proof. Consider the following Markov Decision Process (MDP) with set of states $\Omega$. Player 0 acts as the decision maker. The process that drives the transition to a new state from $\omega$ to $\omega^{\prime}$, given choice $z$, is $Q\left(\omega^{\prime} \mid \omega, z\right)$. Rewards in state $\omega=(x, i) \in \Omega$ are given by $\pi_{0}(x)$ and the discount factor is equal to $\delta$. We assume that in every state $(x, i) \in \Omega$ for which $i \neq 0$, nature chooses $b_{i}(x)$. A policy of the decision maker is a function $\tilde{p}: H_{0} \rightarrow X_{0}$ and a stationary policy of the decision maker is represented by a function $p: X \rightarrow X_{0}$. The collection of policies is denoted by $\tilde{P}$ and the collection of stationary policies by $P$.

According to the standard theory of MDPs, see for instance Puterman (1994), the MDP has an optimal policy which is stationary, say $p^{*} \in P$. Since the reward at history $h \in H_{0}$ under the policy $\tilde{p} \in P$ is defined as

$$
V_{\tilde{p}}(h)=U_{0}\left(h,\left(\tilde{p}, \tilde{b}_{I}\right)\right),
$$

we have that $p^{*}$ achieves a total discounted reward equal to

$$
\max _{\tilde{p} \in \tilde{P}} V_{\tilde{p}}(h)=\max _{\tilde{s}_{0} \in \tilde{S}_{0}} U_{0}\left(h,\left(\tilde{s}_{0}, \tilde{b}_{I}\right)\right) .
$$

It follows that player 0 has no profitable deviation from $p^{*}$. Since the players in $I$ have no profitable deviation from $\tilde{b}_{I}$, it follows that $\left(p^{*}, b_{I}\right) \in S$ is an SSPE.

Proposition 2.5 makes use of the fact that the optimal behavior of a myopic player $i \in I$ is to use strategy $b_{i}$, independent of the strategy used by other players. Therefore, we can model the decision problem of the farsighted player as an MDP with the decisions by the myopic players treated as moves by nature according to $b_{I}$. It is well-known that there exists an optimal policy in this MDP which is 
stationary. Such an optimal stationary policy together with the strategies $b_{I}$ constitutes an SSPE of $\Gamma^{\prime}$. Note that if $\delta$ is small, we have a unique SPE equal to $b_{I_{0}}$ and therefore a unique SSPE. In this paper, we are mainly interested in the case where $\delta$ is close to 1 .

Proposition 2.6 shows that every SPE yields the same expected utility for player 0, conditional on reaching any history $h \in H$.

Proposition 2.6. For any two SPE strategy profiles $\tilde{r}, \tilde{s} \in \tilde{S}$ of game $\Gamma^{\prime}$ it holds that, for every $h \in H$,

$$
U_{0}(h, \tilde{r})=U_{0}(h, \tilde{s}) .
$$

Proof. Let $\tilde{r}, \tilde{s} \in \tilde{S}$ be two SPE strategy profiles. By Proposition 2.3 it holds that $\tilde{r}_{I}=\tilde{s}_{I}=\tilde{b}_{I}$. It holds that $\tilde{r}_{0}$ and $\tilde{s}_{0}$ are both optimal policies of the MDP constructed in the proof of Proposition 2.5 Therefore, it holds for every $h \in H$ that

$$
U_{0}(h, \tilde{r})=V_{\tilde{r}_{0}}(h)=V_{\tilde{s}_{0}}(h)=U_{0}(h, \tilde{s}) .
$$

Propositions 2.5 and 2.6 establish the existence of an SSPE and the uniqueness of SPE payoffs of the farsighted player for any network structure. Proposition 2.6 implies that there is no loss of generality in payoff terms to only consider stationary strategies as far as the farsighted player is concerned. Note, however, that the myopic players' payoffs may not be the same in each SSPE as the farsighted player might have multiple best strategies that are payoff equivalent for him, but not for the myopic players.

\section{Absorbing effort profiles}

In this section we define absorbing effort profiles, consider their relation to Nash equilibria of the static game $\Gamma$ and show the existence of absorbing effort profiles.

We start out by defining two standard concepts of graph theory, cliques and independent sets, as well as a useful tool for our analysis, called pillar sets.

Definition 3.1 (Cliques). A non-empty set of players $J \subseteq I_{0}$ is a clique if for every $i, j \in J$ we have $g_{i j}=1$.

Every single player constitutes a clique as does every pair of neighbors.

Definition 3.2 (Independent sets). Let $J \subseteq I_{0}$. The set of players $K \subseteq J$ is an independent set of $J$ if for every $i, j \in K$ with $i \neq j$ it holds that $g_{i j}=0$. The set $M \subseteq J$ is a maximal independent set of $J$ if it is an independent set of $J$ and $J \subseteq \bar{N}_{M}$. The set $M \subseteq J$ is a maximum independent set of $J$ if it is an independent set of $J$ and there is no other independent set $K$ of $J$ for which $|K|>|M|$.

Notice that for every $J \subseteq I_{0}$, every maximum independent set of $J$ is also a maximal independent set of $J$. 
We introduce pillar sets to serve as an upper bound on the total efforts of a subset of myopic players. The idea is the following: We pick a myopic player with positive effort, called a pillar, whose neighborhood without the farsighted player has a total effort of at most one. We remove the player and his myopic neighbors from the network. We find another such player and repeat until we run out of players with positive effort. The pillar set contains the players selected by this process. The name pillar is chosen for the players' property of supporting at most 1 unit of effort. For an effort profile $x \in X$, we let $I^{+}(x)=\left\{i \in I: x_{i}>0\right\}$ and $I^{0}(x)=\left\{i \in I: x_{i}=0\right\}$ denote the myopic contributors and free riders in effort profile $x$, respectively.

Definition 3.3 (Pillar set). Let $J \subseteq I$ and $x \in X$. The maximal independent set $P$ of $J \cap I^{+}(x)$ is a pillar set of $J$ in $x$ if there exists an enumeration of the elements of $P,\left(i^{1}, \ldots, i^{|P|}\right)$, such that for every $k^{\prime} \in\{1, \ldots,|P|\}$ we have $x\left(\left(J \cap \bar{N}_{i^{k^{\prime}}}\right) \backslash \bigcup_{k=1}^{k^{\prime}-1} \bar{N}_{i^{k}}\right) \leq 1$.

We denote the collection of pillar sets of $J$ in effort profile $x$ by $\mathcal{P}_{x}(J)$. Pillar sets may not exist, for instance when every player has an effort level exceeding 1 , in which case $\mathcal{P}_{x}(J)=\emptyset$. In case $J \cap I^{+}(x)=\emptyset$, it holds that $P=\emptyset$ is a pillar set and $\mathcal{P}_{x}(J)=\{\emptyset\}$. The cardinality of a pillar set $P \in \mathcal{P}_{x}(J)$ provides an upper bound on the total effort $x(J)$ exerted by the players in the set $J$.

By Definition 3.3. two properties immediately follow.

1. Inclusion. Let $J, J^{\prime} \subseteq I$ with $J \subseteq J^{\prime}$ and $x \in X$ be given. If $P \in \mathcal{P}_{x}\left(J^{\prime}\right)$ and $P \subseteq J$, then $P \in \mathcal{P}_{x}(J)$.

2. Addition. Let $J, J^{\prime} \subseteq I$ and $x \in X$ be given. If $P \in \mathcal{P}_{x}(J), P^{\prime} \in \mathcal{P}_{x}\left(J^{\prime}\right)$, and $P \cup P^{\prime}$ is an independent set, then $P \cup P^{\prime} \in \mathcal{P}_{x}\left(J \cup J^{\prime}\right)$.

Let $X^{\prime} \subseteq X$ denote the set of effort profiles that accommodate a pillar set for every $J \subseteq I$, so $\mathcal{P}_{x}(J) \neq \emptyset$ for every $J \subseteq I$. It is possible to give examples such that $\mathcal{P}_{x}(I) \neq \emptyset$, whereas for some $J \subseteq I$ it holds that $\mathcal{P}_{x}(J)=\emptyset$. For instance, for the four-player line network and effort profile $x$ in Figure $3, P=\{1,3\}$ is a pillar set of $I=\{1,2,3\}$ in $x$ when using the enumeration $(1,3)$, but there is no pillar set of $J=\{2,3\} \subset I$ in $x$. Since the effort of the farsighted player does not matter for the definition of a pillar set, we can write $X^{\prime}=X_{0} \times X_{I}^{\prime}$. Notice that $X_{I}^{\prime}$ is a finite set.

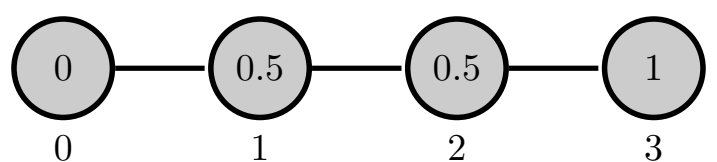

Figure 3: Example of an effort profile that accommodates a pillar set for the full set of myopic players, but not for some subset of it.

Proposition 3.5 states that if every myopic player $i \in I$ uses the best-responder strategy $b_{i}$, then the set $X^{\prime}$ is reachable from any initial state. Moreover, effort levels will stay within the set $X^{\prime}$ once it is reached. We next give a formal definition of reachability. 
Definition 3.4 (Reachability). Let $x, x^{\prime} \in X$ and $s \in S$. Effort profile $x^{\prime}$ is reachable from $x$ in $s$ if there is $T \in \mathbb{N}$ and a $T$-long history $h=\left(\omega^{1}, z^{1}, \omega^{2}, z^{2}, \ldots, \omega^{T-1}, z^{T-1}, \omega^{T}\right) \in H^{T}$ with $x^{0}=x$ and $x^{T-1}=x^{\prime}$ and updates are made in accordance with strategy profile $s$, i.e., for every $t \in\{1, \ldots, T-1\}$, $z^{t}=s_{i^{t}}\left(x^{t-1}\right)$.

If $x^{\prime}$ is reachable from $x$ in $s$, then we write $x \precsim_{s} x^{\prime}$. We write $x \sim_{s} x^{\prime}$ if $x \precsim_{s} x^{\prime}$ and $x^{\prime} \precsim_{s} x$ both hold and $x \prec_{s} x^{\prime}$ if $x \precsim_{s} x^{\prime}$ and $x^{\prime} \mathcal{L}_{s} x$. Clearly, $\sim_{s}$ is an equivalence relation. An equivalence class of $\sim_{s}$ containing $x$ is a recurring set in $s$ if there exists no $x^{\prime} \in X$ for which $x \prec x^{\prime}$. The recurring set in $s$ that contains the effort profile $x \in X$ is denoted by $[x]_{s}$. If an effort profile $y \in X$ belongs to $[x]_{s}$ for some $x \in X$, then we say that $y$ is recurring. Let $R_{s}(x)=\left\{x^{\prime} \in X: x \precsim s x^{\prime}\right\}$ denote the set of effort profiles that are reachable from $x$ in $s$. For $J \subseteq I_{0}$, let $R_{s}^{J}(x) \subseteq R_{s}(x)$ denote the set of effort profiles that are reachable from $x$ in $s$ if updates are restricted to the players in $J$.

Proposition 3.5 (Recurrent effort profiles admit pillar sets.). Let $s \in S_{0} \times\left\{b_{I}\right\}$.

1. Reachability: For every $x \in X \backslash X^{\prime}$ it holds that $R_{s}^{I}(x) \cap X^{\prime} \neq \emptyset$.

2. No exit: For every $x \in X^{\prime}$ it holds that $R_{s}(x) \subseteq X^{\prime}$.

Proof. To prove Reachability, we show that once every myopic player has been active at least once, the resulting effort profile must be in $X^{\prime}$. Let $\left(\left(x^{0}, 1\right), b_{1}\left(x^{0}\right), \ldots,\left(x^{n-1}, n\right), b_{n}\left(x^{n-1}\right),\left(x^{n}, i^{n+1}\right)\right) \in H^{n+1}$ be such that $x^{0} \in X \backslash X^{\prime}$. The proof constructs a pillar set of $x^{n}$ for an arbitrary $J \subseteq I$.

Fix $J \subseteq I$. If $J \cap I^{+}\left(x^{n}\right)=\emptyset$, then the empty set is a pillar set of $J$ in $x^{n}$. Otherwise, let $j_{1}$ be the highest numbered player in $J \cap I^{+}\left(x^{n}\right)$. Since $x_{j_{1}}^{j_{1}}=b_{j_{1}}\left(x^{j_{1}-1}\right)$ and $x_{j_{1}}^{j_{1}}>0$, it holds that $c_{j_{1}}\left(x^{j_{1}}\right)=1$. Since $j_{1}$ is the highest numbered player in $J \cap I^{+}\left(x^{n}\right)$, we have

$$
1=c_{j_{1}}\left(x^{j_{1}}\right) \geq c_{j_{1}}\left(x^{n}\right)=x^{n}\left(\bar{N}_{j_{1}}\right) \geq x^{n}\left(J \cap \bar{N}_{j_{1}}\right) .
$$

If $J \backslash \bar{N}_{j_{1}}=\emptyset$, then it holds that $\left\{j_{1}\right\} \in \mathcal{P}_{x^{n}}(J)$. Otherwise, let $j_{2}$ be the highest numbered player in $\left(J \backslash \bar{N}_{j_{1}}\right) \cap I^{+}\left(x^{n}\right)$. It holds that

$$
1=c_{j_{2}}\left(x^{j_{2}}\right)=x^{j_{2}}\left(\bar{N}_{j_{2}}\right) \geq x^{j_{1}}\left(\left(J \cap \bar{N}_{j_{2}}\right) \backslash \bar{N}_{j_{1}}\right) \geq x^{n}\left(\left(J \cap \bar{N}_{j_{2}}\right) \backslash \bar{N}_{j_{1}}\right) .
$$

If $J \backslash\left(\bar{N}_{j_{1}} \cup \bar{N}_{j_{2}}\right)=\emptyset$, then it holds that $\left\{j_{1}, j_{2}\right\} \in \mathcal{P}_{x^{n}}(J)$. Otherwise, we continue as before and find a pillar set $\left\{j_{1}, \ldots, j_{k}\right\} \in \mathcal{P}_{x^{n}}(J)$ in $k \leq n$ steps.

We now prove the No exit part. Suppose, by contradiction, that there exists $T \in \mathbb{N}$ and a $(T+1)$ long history $h=\left(\omega^{1}, z^{1}, \ldots, \omega^{T}, z^{T}, \omega^{T+1}\right) \in H^{T+1}$ with $x^{0} \in X^{\prime}$ and $x^{T} \in X \backslash X^{\prime}$. Since $x^{T} \in X \backslash X^{\prime}$, there exists $J \subseteq I$ such that $\mathcal{P}_{x^{T}}(J)=\emptyset$.

First, consider the case $|J|=1$, i.e., $J=\{j\}$. If $x_{j}^{T}=0$, then $\emptyset \in \mathcal{P}_{x^{T}}(J)$, leading to a contradiction. If $0<x_{j}^{T} \leq 1$, then $\{j\} \in \mathcal{P}_{x^{T}}(J)$, leading to a contradiction. If $x_{j}^{T}>1$ and $j \notin\left\{i^{1}, \ldots, i^{T}\right\}$, then $x_{j}^{0}=x_{j}^{T}>1$, so $\mathcal{P}_{x^{0}}(\{j\})=\emptyset$, a contradiction to $x^{0} \in X^{\prime}$. If $x_{j}^{T}>1$ and $j \in\left\{i^{1}, \ldots, i^{T}\right\}$, then $x_{j}^{T} \in[0,1]$, a contradiction to $x_{j}^{T}>1$. 
Now assume $|J|=k$ for some $k>1$ and for every $J^{\prime} \subseteq I$ with $\left|J^{\prime}\right|<k$ it holds that $\mathcal{P}_{x^{T}}\left(J^{\prime}\right) \neq \emptyset$. If there exists $j \in J$ with $x_{j}^{T}=0$, then consider $J^{\prime}=J \backslash\{j\}$. We have that $\mathcal{P}_{x^{T}}(J)=\mathcal{P}_{x^{T}}\left(J^{\prime}\right) \neq \emptyset$, where we use that $\left|J^{\prime}\right|<k$, leading to a contradiction. Consequently, it holds that $J \subseteq I^{+}\left(x^{T}\right)$.

If $J \cap\left\{i^{1}, \ldots, i^{T}\right\}=\emptyset$, then for every $j \in J$ we have $x_{j}^{0}=x_{j}^{T}$. It follows that $\emptyset \neq \mathcal{P}_{x^{0}}(J)=\mathcal{P}_{x^{T}}(J)=$ $\emptyset$, a contradiction. Consequently, it holds that $J \cap\left\{i^{1}, \ldots, i^{T}\right\} \neq \emptyset$.

Let $j$ be the last player in $\left(i^{1}, \ldots, i^{T}\right)$ who belongs to $J$. By this choice, we must have $x^{T}\left(\bar{N}_{j} \cap J\right) \leq 1$. Consider the set of players $J^{\prime}=J \backslash \bar{N}_{j}$. Since $\left|J^{\prime}\right|<k$, it holds that $\mathcal{P}_{x^{T}}\left(J^{\prime}\right) \neq \emptyset$. Let $P^{\prime} \in \mathcal{P}_{x^{T}}\left(J^{\prime}\right)$. Then, since $P^{\prime} \cap \bar{N}_{j}=\emptyset$, by the addition property of pillar sets, we have $P^{\prime} \cup\{j\} \in \mathcal{P}_{x^{T}}(J)$, which leads to a contradiction.

Remark 3.6. By the proof of Proposition 3.5, two statements follow:

1. The set $X^{\prime}$ is reachable from any effort profile via at most $n$ updates, hence it will be reached almost surely for any starting state.

2. For a fixed $x_{0} \in X_{0}$, once the game reaches $\left\{x_{0}\right\} \times X_{I}^{\prime}$, it cannot leave this set unless player 0 updates. The proof is identical to that of the No exit part of Proposition 3.5 .

As a consequence of Proposition 3.5, for any strategy profile $s \in S_{0} \times\left\{b_{I}\right\}$, we must have $[x]_{s} \subseteq X^{\prime}$.

Let

$$
X^{*}=\left\{x \in X \mid \forall i \in I, x_{i}=b_{i}(x)\right\}
$$

denote the set of effort profiles where every myopic player $i \in I$ is at his best response, so $c_{i}(x)=1$ if $x_{i}>0$, and $c_{i}(x) \geq 1$ if $x_{i}=0$. The set $X^{*}$ contains all Nash equilibria of the static game $\Gamma$.

Definition 3.7 (Absorbing effort profile). Let $s_{0} \in S_{0}$. An effort profile $x \in X$ is absorbing for $s_{0}$ if $x \in X^{*}$ and $s_{0}(x)=x_{0}$. The set of absorbing effort profiles for $s_{0}$ is denoted by $A\left(s_{0}\right)$.

In other words, an effort profile is absorbing for a given strategy $s_{0} \in S_{0}$ if every myopic player is at his best response and the farsighted player also does not deviate from it. Clearly, we have $A\left(s_{0}\right) \subseteq X^{*}$. It is also easily observed that $A\left(b_{0}\right)$ corresponds to the set of Nash equilibria of the static game $\Gamma$.

We now identify a profile $x^{*} \in X$ that is absorbing for every SSPE. Let $M$ be a maximum independent set of $N_{0}$. Let $M^{\prime} \supseteq M$ be a maximal independent set of $I_{0}$. Such a set $M^{\prime}$ can be easily constructed, by adding a player who is not in $\bar{N}_{M}$ to $M$, then adding a player outside the neighborhood of $M$ and the new player, and so on, until we run out of players. Let $x^{M^{\prime}} \in X$ be defined as $x_{i}^{M^{\prime}}=1$ if $i \in M^{\prime}$ and $x_{i}^{M^{\prime}}=0$ if $i \in I_{0} \backslash M^{\prime}$. Notice that $N_{0} \neq \emptyset$ implies $0 \notin M^{\prime}$ and $N_{0}=\emptyset$ implies $0 \in M^{\prime}$. Let $X^{\text {a }}$ be the non-empty set of effort profiles $x^{M^{\prime}}$ that can be constructed in this way, i.e., the set of effort profiles $x^{M^{\prime}}$ such that $M^{\prime}$ is a maximal independent set of $I_{0}$ and $M^{\prime} \cap N_{0}$ is a maximum independent set of $N_{0}$.

Proposition 3.8 (Existence of absorbing effort profiles). For every SSPE $s^{*} \in S^{*}$ it holds that $X^{\mathrm{a}} \subseteq$ $A\left(s_{0}^{*}\right)$. Moreover, it holds that $X^{\mathrm{a}} \subseteq A\left(b_{0}\right)$. 
Proof. Assume $N_{0}=\emptyset$. Let $M^{\prime}$ be a maximal independent set of $I_{0}$. It holds that $x_{0}^{M^{\prime}}=1$. Since $N_{0}=\emptyset$, player 0 achieves the highest possible instantaneous payoff at $x^{M^{\prime}}$. It also holds that $\pi_{0}(x)<\pi_{0}\left(x^{M^{\prime}}\right)$ whenever $x_{0} \neq 1$. It follows that for every $s^{*} \in S^{*}$ it holds that $s_{0}^{*}\left(x^{M^{\prime}}\right)=x_{0}^{M^{\prime}}=1$. Consider a player $j \in I$ with $x_{j}^{M^{\prime}}=1$. By the definition of an independent set, it holds that $x_{i}=0$ for every $i \in N_{j}$, so $b_{j}\left(x^{M^{\prime}}\right)=x_{j}^{M^{\prime}}=1$. Finally, consider a player $j \in I$ with $x_{j}^{M^{\prime}}=0$. Since $M^{\prime}$ is a maximal independent set, it holds that $j \in N_{M^{\prime}}$, so there is $i \in N_{j}$ with $x_{i}^{M^{\prime}}=1$. It follows that $b_{j}\left(x^{M^{\prime}}\right)=x_{j}^{M^{\prime}}=0$. We have shown that $x^{M^{\prime}} \in A\left(s_{0}^{*}\right)$. Since $b_{0}\left(x^{M^{\prime}}\right)=x_{0}^{M^{\prime}}=1$, we also have $x^{M^{\prime}} \in A\left(b_{0}\right)$.

Assume $N_{0} \neq \emptyset$. Let $M$ be a maximum independent set of $N_{0}$ and $M^{\prime} \supseteq M$ be a maximal independent set of $I_{0}$. It holds that $x_{0}^{M^{\prime}}=0$. Since $x^{M^{\prime}} \in X^{\prime}$, it holds by Proposition 3.5 that $R_{s^{*}}\left(x^{M^{\prime}}\right) \subseteq X^{\prime}$. Since the cardinality of a pillar set of $N_{0}$ is an upper bound on the total effort as made by the players in $N_{0}$ and $M$ is a pillar set of $N_{0}$ with the highest cardinality, it holds that, for every $x \in X^{\prime}$, $\pi_{0}(x) \leq \pi_{0}\left(x^{M^{\prime}}\right)$. It also holds that $\pi_{0}(x)<\pi_{0}\left(x^{M^{\prime}}\right)$ whenever $x_{0}>0$. It follows that, for every $s^{*} \in S^{*}, s_{0}^{*}\left(x^{M^{\prime}}\right)=x_{0}^{M^{\prime}}=0$. Consider a player $j \in I$ with $x_{j}^{M^{\prime}}=1$. By the definition of an independent set, it holds that $x_{i}=0$ for every $i \in N_{j}$, so $b_{j}\left(x^{M^{\prime}}\right)=x_{j}^{M^{\prime}}=1$. Finally, consider a player $j \in I$ with $x_{j}^{M^{\prime}}=0$. Since $M$ is a maximal independent set, it holds that $j \in N_{M^{\prime}}$, so there is $i \in N_{j}$ with $x_{i}^{M^{\prime}}=1$. It follows that $b_{j}\left(x^{M^{\prime}}\right)=x_{j}^{M^{\prime}}=0$. We have shown that $x^{M^{\prime}} \in A\left(s_{0}^{*}\right)$. Since $b_{0}\left(x^{M^{\prime}}\right)=x_{0}^{M^{\prime}}=0$, we also have $x^{M^{\prime}} \in A\left(b_{0}\right)$.

Proposition 3.8 identifies an effort profile which is both acceptable for the myopic players, as they are playing best responses, and for the farsighted player, who gets the maximum instantaneous payoff attainable from this position. By this construction we show not only that the set of absorbing effort profiles is non-empty for every SSPE of $\Gamma^{\prime}$, but also that it has a non-empty intersection with the set of Nash equilibria of the static game $\Gamma$. The next two examples show that the two sets do not generally include each other.

In Example 3.9 it holds that $A\left(b_{0}\right) \nsubseteq A\left(s_{0}^{*}\right)$.

Example 3.9. Consider the kite network presented in the introduction. Let $I_{0}=\{0,1,2,3\}, g_{01}=$ $g_{02}=g_{03}=g_{12}=g_{23}=1$, and $g_{13}=0$ as shown in Figure 1. We can take any $\left(f_{i}\right)_{i \in I_{0}},\left(\gamma_{i}\right)_{i \in I_{0}}$, and $d$ satisfying the assumptions of Section 2. We claim that $A\left(b_{0}\right) \nsubseteq A\left(s_{0}^{*}\right)$ for a high enough $\delta$. It is clear that $A\left(b_{0}\right)$ includes the profile $(0,0,1,0)$ but, as it will be apparent, $A\left(s^{*}\right)$ does not.

By Proposition 3.8, $x^{*}=(0,1,0,1)$ is absorbing in every SSPE. We argue that player 0 has a strategy to ensure that $x^{*}$ is reached almost surely, irrespective of the initial effort profile $x^{0}$, after which neither the farsighted player nor the myopic opponents will be better off by moving away. The strategy $s_{0}^{\prime} \in S_{0}$ is defined by setting, for $x \in X$,

$$
s_{0}^{\prime}(x)= \begin{cases}0 & \text { if }\left(x_{1}, x_{2}, x_{3}\right) \in\{(0,0,0),(1,0,0),(0,0,1),(1,0,1)\} \\ 1 & \text { otherwise }\end{cases}
$$

A typical play of the game with the strategy profile $\left(s_{0}^{\prime}, b_{1}, b_{2}, b_{3}\right)$ proceeds as follows. If the game begins in a generic effort profile, the farsighted player sets his own effort level to 1 as soon as he receives a 
revision opportunity. Afterwards, every time a myopic player receives a revision opportunity, his efforts are set equal to 0 , reaching the profile $(1,0,0,0)$. Once this is achieved, the farsighted player chooses effort level 0 . If player 2 receives the next revision opportunity, he chooses effort level 1 , reaching the profile $(0,0,1,0)$, where all myopic players are at best response, so only by player 0 's revision will the game leave this profile, resetting the game to the effort profile $(1,0,0,0)$, and eventually to $(0,0,0,0)$. If player 0 receives a revision opportunity at $(0,0,0,0)$, nothing happens. If either player 1 or player 3 receives the revision opportunity, then the game necessarily progresses to the absorbing profile $(0,1,0,1)$.

Starting from initial effort profile $x^{0}=(0,0,1,0)$, strategy $s_{0}^{\prime}$ almost surely leads to effort profile $(0,1,0,1)$, and, once this effort profile is reached, stays there and player 0 has a consumption equal to 2 in every period without making any effort. It follows that for a sufficiently high $\delta$ no SSPE $s^{*} \in S^{*}$ can be such that $s_{0}^{*}(0,0,1,0)=0$, since then consumption of the farsighted player would be equal to 1 in every period. We have that $(0,0,1,0) \notin A\left(s_{0}^{*}\right)$, whereas clearly $(0,0,1,0) \in A\left(b_{0}\right)$.

In Example 3.10 it holds that $A\left(s_{0}^{*}\right) \nsubseteq A\left(b_{0}\right)$.

Example 3.10. Let $I_{0}=\{0,1,2\}$, for every $i \in I_{0}, \gamma_{i}=1$, and $d=2$. We can take any $\left(f_{i}\right)_{i \in I_{0}}$ satisfying the assumptions of Section 2. The network $G$ is given by $g_{01}=g_{12}=1$ and $g_{02}=0$ and is illustrated in Figure 4. Consider the effort profile $x^{0}=(0,0.5,0.5)$ (see the left side of Figure 4). Since $b_{0}\left(x^{0}\right)=1 / 2$, it is clear that $x^{0} \notin A\left(b_{0}\right)$. We claim that $x^{0} \in A\left(s^{*}\right)$ for a sufficiently high $\delta$.
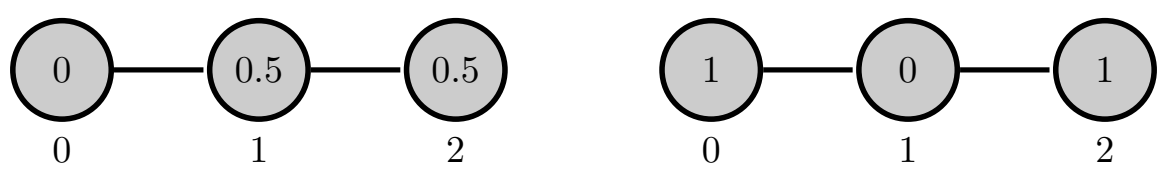

\footnotetext{
Figure 4: The network and initial effort profile of Example 3.10 Player 0 can choose between remaining under his target consumption (left), or increasing his efforts and prompting a response (right). If player 0 increases his effort, he faces a permanent decrease in efforts from player 1, who free rides on the effort exerted by player 2 . There is nothing player 0 can do to change that.
}

Clearly, for a strategy $s_{0} \in S_{0}$ such that $s_{0}\left(x^{0}\right)=0$, it holds that $x^{0} \in A\left(s_{0}\right)$, whereas $x^{0}$ is not a Nash equilibrium of the static game $\Gamma$. By playing 0 at $x^{0}$, player 0 receives an indefinite payoff stream equal to $f_{0}(0.5)$. Now consider $s_{0} \in S_{0}$ with $s_{0}\left(x^{0}\right)>0$. The only effort profiles $x \in X$ that can be reached from $x^{0}$ are the ones where $\left(x_{1}, x_{2}\right)=(1 / 2,1 / 2)$ or $\left(x_{1}, x_{2}\right)=(0,1)$. Moreover, almost surely an effort profile with $\left(x_{1}, x_{2}\right)=(0,1)$ is reached, since if players 1 and 2 receive revision opportunities in this order, they will set their efforts to 0 and 1, respectively. From this point onwards, by the assumptions on $f_{0}$, every period's instantaneous payoff that player 0 receives is at most $f_{0}(1)-1$. Hence, if $f_{0}(1)-1<f_{0}(0.5)$ and if $\delta$ is sufficiently high, it holds that $s_{0}^{*}\left(x^{0}\right)=0$ for every SSPE $s^{*} \in S^{*}$.

Notice that the best effort profile for the farsighted player, $x^{*}=(0,1,0)$, cannot be reached when $x^{0}=(0,0.5,0.5)$. 


\section{Dynamic properties of the game}

In this section we discuss the dynamic development of effort levels of the myopic players. We show that repeated updating by a group of myopic players results in reaching a partial Nash equilibrium. We go on to demonstrate that as a result of the local substitution between the efforts of two myopic neighbors, the game has a tendency towards rising inequality of exerted efforts. Players with high consumption become free riders and players with low consumption end up making higher efforts. Finally, we show that players with a distance of at least three from the farsighted player are not affected by him in the long-run, meaning that any effect he might have on the game is either short-lived or localized. We show a similar result for particular sets of contributing players with consumption equal to one, called factions, that are outside the neighborhood of the farsighted player.

To show that repeated updating by a group of myopic players results in a partial Nash equilibrium, we need the notion of a best-response potential game (Voorneveld, 2000).

Definition 4.1 (Best-response potential game). The static game $\Gamma$ is a best-response potential game if there exists a function $\phi: X \rightarrow \mathbb{R}$ such that, for every $i \in I_{0}$, for every $x \in X$, it holds that

$$
\underset{x_{i} \in X_{i}}{\operatorname{argmax}} \pi_{i}\left(x_{i}, x_{-i}\right)=\underset{x_{i} \in X_{i}}{\operatorname{argmax}} \phi\left(x_{i}, x_{-i}\right) .
$$

The function $\phi$ is called a best-response potential of $\Gamma$.

Proposition 4.2. The function $\phi$ defined by

$$
\phi(x)=\sum_{i \in I_{0}}\left(x_{i}-\frac{1}{2} \sum_{j \in \bar{N}_{i}} x_{i} x_{j}\right), \quad x \in X,
$$

is a best-response potential of $\Gamma$.

Proof. Let some $i \in I_{0}$ and some $x \in X$ be given. We first determine $\operatorname{argmax}_{x_{i} \in \mathbb{R}_{+}} \phi\left(x_{i}, x_{-i}\right)$. Taking the derivative of $\phi$ with respect to $x_{i}$ yields

$$
\frac{\partial \phi\left(x_{i}, x_{-i}\right)}{\partial x_{i}}=1-\frac{1}{2} \sum_{j \in \bar{N}_{i}} x_{j}-\frac{1}{2} \sum_{j \in \bar{N}_{i}} x_{j}=1-\sum_{j \in \bar{N}_{i}} x_{j} .
$$

It therefore holds that

$$
\underset{x_{i} \in \mathbb{R}_{+}}{\operatorname{argmax}} \phi\left(x_{i}, x_{-i}\right)=\max \left\{0,1-\sum_{j \in \bar{N}_{i}} x_{j}\right\} .
$$

Since $x \in X$, it follows that $\max \left\{0,1-\sum_{j \in \bar{N}_{i}} x_{j}\right\}$ is a non-negative multiple of $1 / d$, so belongs to $X_{i}$, and therefore

$$
\underset{x_{i} \in X_{i}}{\operatorname{argmax}} \phi\left(x_{i}, x_{-i}\right)=\max \left\{0,1-\sum_{j \in \bar{N}_{i}} x_{j}\right\} .
$$

Since the right-hand size is equal to $b_{i}(x)$, we have shown that

$$
\underset{x_{i} \in X_{i}}{\operatorname{argmax}} \pi_{i}\left(x_{i}, x_{-i}\right)=\underset{x_{i} \in X_{i}}{\operatorname{argmax}} \phi\left(x_{i}, x_{-i}\right) .
$$


This result is analogous to ones in Bramoullé et al. (2014), who show that $\phi$ is an exact potential of a game with linear-quadratic payoffs, and in Bayer et al. (2019), who show that it is a best-response potential of weighted network games where the strategies are on a continuum. Parise and Özdaglar (2019) generalize this result by the use of variational inequalities for a wider class of games.

We can use Proposition 4.2 to show that repeated updating by a group of myopic players reaches an effort profile where they all play best responses, thereby reaching a partial Nash equilibrium.

Proposition 4.3 (Reaching a partial Nash equilibrium). Let $s \in S_{0} \times\left\{b_{I}\right\}$ and $J \subseteq I$. For every $x \in X$ there exists $y \in R_{s}^{J}(x)$ such that, for every $j \in J, y_{j}=b_{j}(y)$.

Proof. Let some $x \in X$ be given and let all players in $J$ update once in some given order, leading to effort profile $x^{1}$. For every $j \in J$, it holds that $x_{j}^{1} \leq 1$. Any effort profile in $R_{s}^{J}\left(x^{1}\right)$ has effort levels by players in $J$ between 0 and 1 , so $R_{s}^{J}\left(x^{1}\right)$ is a finite set. Either, for every $j \in J, x_{j}^{1}=b_{j}(x)$ and we are done, or there is $j \in J$ such that $x_{j}^{1} \neq b_{j}\left(x^{1}\right)$. In the latter case, an update by player $j$ results in $x^{2}=\beta_{j}\left(x^{1}\right)$. It holds that $\phi\left(x^{2}\right)>\phi\left(x^{1}\right)$. Either, for every $j \in J, x_{j}^{2}=b_{j}(x)$ and we are done, or there is $j \in J$ such that $x_{j}^{2} \neq b_{j}\left(x^{2}\right)$. Proceeding in this way, the fact that $R_{s}^{J}\left(x^{1}\right)$ is a finite set and the fact that $\phi$ strictly increases in each iteration implies that we find $y \in R_{s}^{J}(x)$ in a finite number of steps.

Since only players in $J$ are updating in the statement of Proposition 4.3, it follows that, for every $i \in I_{0} \backslash J, y_{i}=x_{i}$. Proposition 4.3 therefore means that updating by a group of myopic players leads to a partial Nash equilibrium, irrespective of the effort levels of the players not belonging to the group. A particular special case results when $J=I$. If the farsighted player's efforts are held constant - whether by his own choice or by the fact that he is not selected to become active for a time - the myopic players will reach a partial Nash equilibrium of the game where the effort level of the farsighted player has been fixed.

We show that these dynamics lead to a rise of inequality in the efforts exerted to create the local public good.

Proposition 4.4 (Rising inequality). Let $s \in S_{0} \times\left\{b_{I}\right\}$. Let $x \in X, i \in I$, and $J \subseteq I \cap N_{i}$ be given such that for every $j \in J$ it holds that $c_{j}(x)>c_{i}(x)$. Then there exists $y \in R_{s}^{J \cup\{i\}}(x)$ such that $y(J)=0$ and $y_{i} \geq x_{i}$.

Proof. First we show the statement for $|J|=1$, so $J=\{j\}$. If $x_{j}=0$, the statement is true. If $x_{j}>0$ and $x\left(N_{j}\right) \geq 1$, then the profile $y=\beta_{j}(x)$ satisfies all the desired properties.

We now consider the case where $x_{j}>0$ and $x\left(N_{j}\right)<1$. Let players $j$ and $i$ revise once in sequence and denote the resulting profile by $x^{\prime}$. It holds that $c_{j}\left(x^{\prime}\right)>c_{i}\left(x^{\prime}\right)$ as only the neighbors $i$ and $j$ updated. Additionally, we have $x_{j}^{\prime}=b_{j}(x)=1-x\left(N_{j}\right)>0$ and $x_{i}^{\prime}=b_{i}\left(x^{\prime}\right)=\max \left\{0,1-x\left(N_{i}\right)+x_{j}-x_{j}^{\prime}\right\}$. Substituting for $x_{j}^{\prime}$ we get

$$
x_{i}^{\prime}=\max \left\{0, x\left(N_{j}\right)-x\left(N_{i}\right)+x_{j}\right\}=\max \left\{0, c_{j}(x)-c_{i}(x)+x_{i}\right\}>x_{i} .
$$

We therefore must have $c_{j}\left(x^{\prime}\right)>c_{i}\left(x^{\prime}\right)=1$ as well. 
Consider a sequence of states $\left(\left(x^{0}, i^{1}\right),\left(x^{1}, i^{2}\right), \ldots,\left(x^{2 d-1}, i^{2 d}\right)\right) \in \Omega^{2 d}$ such that $x^{0}=x^{\prime}, i^{t}=j$ if $t$ is odd and $i^{t}=i$ if $t$ is even, and for every $t \geq 1$ we have $x^{t}=\beta_{i^{t}}\left(x^{t-1}\right)$. Since $c_{j}\left(x^{1}\right)>1$ we have $x_{j}^{1}<x_{j}^{0}$ and $c_{i}\left(x^{1}\right)=1-x_{j}^{0}+x_{j}^{1}$, hence player $i$ will increase his effort by the same amount as player $j$ 's decrease, i.e., $x_{i}^{2}-x_{i}^{1}=x_{j}^{0}-x_{j}^{1}$. Since $c_{j}\left(x^{2}\right)>c_{i}\left(x^{2}\right)=1$ and $x_{i}^{2}>x_{i}^{1}$, the same argument can be repeated. After at most $2 d$ steps, player $j$ will reach 0 and player $i$ will have taken over all of player $j$ 's efforts and the resulting effort profile satisfies all the desired properties.

Now let $J$ be a non-singleton set of myopic players. Fix $j \in J$. As shown above, a sequence of alternating updates by players $j$ and $i$ allows the game to reach an action profile $x^{\prime} \in X$ for which we have $x_{j}^{\prime}=0$ and $x_{i}^{\prime} \geq x_{i}$. We now show that for every $j^{\prime} \in J \backslash\{j\}$ it holds that $c_{j^{\prime}}\left(x^{\prime}\right)>c_{i}\left(x^{\prime}\right)$. We have

$$
\begin{aligned}
c_{j^{\prime}}\left(x^{\prime}\right) & =x^{\prime}\left(\bar{N}_{j^{\prime}} \backslash\{i, j\}\right)+x_{i}^{\prime}+g_{j j^{\prime}} x_{j}^{\prime}=c_{j^{\prime}}(x)+\left(x_{i}^{\prime}-x_{i}\right)+g_{j j^{\prime}}\left(x_{j}^{\prime}-x_{j}\right), \\
c_{i}\left(x^{\prime}\right) & =x^{\prime}\left(\bar{N}_{i} \backslash\{i, j\}\right)+x_{i}^{\prime}+x_{j}^{\prime}=c_{i}(x)+\left(x_{i}^{\prime}-x_{i}\right)+\left(x_{j}^{\prime}-x_{j}\right) .
\end{aligned}
$$

Since $x_{j}^{\prime}-x_{j} \leq 0$ and $g_{j j^{\prime}} \in\{0,1\}$, we have $\left(g_{j j^{\prime}}-1\right)\left(x_{j}^{\prime}-x_{j}\right) \geq 0$, hence we have $c_{j^{\prime}}\left(x^{\prime}\right)-c_{i}\left(x^{\prime}\right) \geq$ $c_{j^{\prime}}(x)-c_{i}(x)>0$.

Therefore, we can proceed as in the singleton case until every player in $J$ exerts an effort of 0 and player $i$ takes over the efforts of every $j \in J$. The resulting profile satisfies all the desired properties.

We now introduce two notions to describe sets of contributing myopic players.

Definition 4.5 (Islands and factions). Let $x \in X$. A non-empty, connected set of myopic players $L \subseteq I$ is an island in $x$ if for every $j \in L$ it holds that $x_{j}>0$ and for every $i \in N_{L} \backslash\{0\}$ it holds that $x_{i}=0$. An island $F \subseteq I$ is a faction in $x$ if for every $j \in F$ it holds that $c_{j}(x)=1$. The collection of islands in $x$ is denoted by $\mathcal{L}(x)$ and the collection of factions by $\mathcal{F}(x)$.

An island is a set of contributing myopic players whose myopic neighbors outside the set are free riders. A faction is an island where every player has a consumption level equal to 1 and is therefore at his best response.

Consider a recurring effort profile and a faction in this effort profile outside the neighborhood of the farsighted player. The next result states that every player in the faction and its neighborhood chooses the same effort level in every effort profile that can be reached.

Proposition 4.6 (Members of factions outside $N_{0}$ never change their efforts). Let $s \in S_{0} \times\left\{b_{I}\right\}$, let $x \in X$ be recurring, and let $y \in[x]_{s}$. For every $F \in \mathcal{F}(x)$ such that $F \cap N_{0}=\emptyset$, for every $j \in \bar{N}_{F}$, it holds that $b_{j}(x)=x_{j}=y_{j}=b_{j}(y)$.

Proof. Let $F \in \mathcal{F}(x)$ be such that $F \cap N_{0}=\emptyset$.

Assume that $|F|=1$, i.e., $F$ is a one-player faction, $\{j\}$. Since $F$ is a faction, it holds that $x_{j}=1$ and, for every $i \in N_{j}, x_{i}=0$. It therefore holds that $b_{j}(x)=1$, whereas for every $i \in N_{j}$ it holds that $b_{i}(x)=0$. Any effort profile $x^{\prime}$ that results after an update from $x$ therefore satisfies $x_{j}^{\prime}=1$ and, for 
every $i \in N_{j}, x_{i}^{\prime}=0$. Iterating this argument yields that $b_{j}(y)=y_{j}=1=x_{j}=b_{j}(x)$ and, for every $i \in N_{j}, b_{i}(y)=y_{i}=0=x_{i}=b_{i}(x)$.

Now let $m>1$ and assume that the statement holds for every faction with fewer than $m$ members. Assume $|F|=m$.

Suppose $y_{j} \neq x_{j}$ for some $j \in \bar{N}_{F}$. Let $i^{1}, \ldots, i^{T}$ be a sequence of updating players in $I_{0}$ and $x^{0}, \ldots, x^{T}$ be a sequence of effort profiles in $X$ such that, for every $t=1, \ldots, T, x^{0}=x, x^{t}=\beta_{i^{t}}\left(x^{t-1}\right)$, and $x^{T}=y$. Let $t$ be the lowest element of $\{1, \ldots, T\}$ for which there is $i \in \bar{N}_{F}$ such that $x_{i}^{t} \neq x_{i}$. Since for every $i \in F$ it holds that $x_{i}=b_{i}(x)$, it follows that $i^{t} \in N_{F}$, so $x_{i^{t}}^{t}>0=x_{i^{t}}$, and, for every $i \in \bar{N}_{F} \backslash\left\{i^{t}\right\}, x_{i}^{t}=x_{i}$.

It holds that $c_{i^{t}}\left(x^{t}\right)=1$ as $x_{i^{t}}=b_{i^{t}}\left(x^{t}\right)>0$. Furthermore, it must be true that $F \backslash N_{i^{t}} \neq \emptyset$, otherwise $x^{t}\left(N_{i^{t}}\right) \geq x^{t}(F) \geq 1$ and $b_{i^{t}}\left(x^{t}\right)=0$, a contradiction. For every $i \in F \backslash N_{i^{t}}$ it holds that $c_{i}\left(x^{t}\right)=c_{i}(x)=1$. Moreover, for every $j \in N_{i^{t}} \cap F$ we have $c_{j}\left(x^{t}\right)=c_{j}(x)+x_{i^{t}}^{t}>1$. Hence, by Proposition 4.4 there exists $y^{1} \in R_{s}^{\left(N_{i^{t}} \cap F\right) \cup\left\{i^{t}\right\}}\left(x^{t}\right)$ such that $y^{1}\left(N_{i^{t}} \cap F\right)=0$. Notice that $i^{t}$ is not in the neighborhood of $F \backslash N_{i^{t}}$, so $\left|F \backslash N_{i^{t}}\right|<m$.

By Proposition 4.3 there exists $y^{2} \in R_{s}^{F \backslash N_{i^{t}}}\left(y^{1}\right)$ such that, for every $i \in F \backslash N_{i^{t}}, y_{i}^{2}=b_{i}\left(y^{2}\right)$. Since $F \cap N_{0}=\emptyset$, there must be a player in $F \backslash N_{i^{t}}$ with positive effort at $y^{2}$, so there is a faction $F^{2} \subseteq F \backslash N_{i^{t}}$ in $y^{2}$. However, since $y^{2} \in[x]_{s}$ and since $\left|F^{2}\right| \leq\left|F \backslash N_{i^{t}}\right|<m$, we must have $x_{i}=y_{i}^{2}$ for every $i \in \bar{N}_{F^{2}}$. This contradicts the assumption that $F \supseteq F^{2}$ is a faction of $m$ players in $x$. Consequently, for every $j \in \bar{N}_{F}$, it holds that $x_{j}=y_{j}$.

Clearly, for every $j \in \bar{N}_{F}$, it holds that $b_{j}(x)=x_{j}$ and $b_{j}(y)=y_{j}$, since otherwise player $j$ would change his effort level when given a revision opportunity.

The intuition behind Proposition 4.6 is as follows. A one-player faction outside player 0's neighborhood will never change as it consists of a myopic player with an effort of 1 surrounded by free riders. None of these players will change their effort levels before the other does so. Suppose the proposition is not true for a two-player faction in a recurrent profile. It can then be shown that it is possible to reach a one-player faction outside player 0's neighborhood. But then the fact that a one-player faction never changes leads to a contradiction with the original two-player faction being recurrent. By induction the same argument can be extended to factions of more players.

The next proposition shows that any non-singleton recurring set contains a profile in which every myopic player is at his best response and every faction is either completely inside or completely outside the farsighted player's neighborhood.

Proposition 4.7 (Factions are either completely inside or completely outside $N_{0}$ ). Let $s \in S_{0} \times\left\{b_{I}\right\}$ and let $x \in X$ be a recurrent profile in $s$. If $\left|[x]_{s}\right|>1$, then there exists $y \in[x]_{s} \cap X^{*}$ such that for every $F \in \mathcal{F}(y)$ it holds that either $F \subseteq N_{0}$ or $F \cap N_{0}=\emptyset$.

Proof. See Appendix. 
Example 3.10 shows that the statement of Proposition 4.7 cannot be extended to singleton recurring sets.

Proposition 4.7 is used to show the next result.

Theorem 4.8 (Local impact only). Let $s \in S_{0} \times\left\{b_{I}\right\}$, let $x \in X$ be recurring, and let $y \in[x]_{s}$. For every $i \in I$ such that $\bar{N}_{i} \cap N_{0}=\emptyset$ it holds that $b_{i}(x)=x_{i}=y_{i}=b_{i}(y)$.

Proof. See Appendix.

Theorem 4.8 states that for every network, all myopic players with a distance of at least three from the farsighted player, i.e. those whose neighborhood does not intersect the neighborhood of the farsighted player, will never change their efforts in a recurring set of effort profiles.

\section{Bounds on payoffs and comparative statics}

We now evaluate networks by comparing two numbers, the lowest and the highest long-run payoff the farsighted player can guarantee for any initial state. We also consider the comparative statics of the bounds as links are added to the network. We therefore make the network explicit in our notation for the game. The collection of networks on the set of players $I_{0}$ satisfying the assumptions of Section 2 is denoted by $\mathcal{G}$, so $\mathcal{G}=\left\{G \in\{0,1\}^{(n+1) \times(n+1)}: \forall i, j \in I_{0}, g_{i j}=g_{j i}, g_{i i}=1\right\}$. For $G \in \mathcal{G}$, let $\mathcal{K}_{0}(G)$ denote the collection of independent sets of $N_{0}(G)$, the myopic players in the farsighted player's neighborhood in network $G$, and let $\bar{e}(G)$ denote the cardinality of a maximum independent set of $N_{0}(G)$, so

$$
\bar{e}(G)=\max _{K \in \mathcal{K}_{0}(G)}|K| .
$$

The number $\bar{e}(G)$ is important for our purposes, since it corresponds to the maximum number of players that the farsighted player can fully exploit simultaneously without any of the myopic players preferring to deviate.

Definition 5.1 (Lower and upper bounds). The lower and upper bounds on the expected payoffs of the farsighted player in the game $\Gamma^{\prime}(G)$ are given by

$$
\begin{aligned}
& \underline{u}(G)=\inf _{\omega \in \Omega, s^{*} \in S^{*}} \lim _{t \rightarrow \infty} u_{0}^{t}\left(\omega, s^{*}\right) . \\
& \bar{u}(G)=\sup _{\omega \in \Omega, s^{*} \in S^{*}} \lim _{t \rightarrow \infty} u_{0}^{t}\left(\omega, s^{*}\right) .
\end{aligned}
$$

We have related the SSPE strategy of the farsighted player to an optimal policy in an MDP, see the proof of Proposition 2.5. Since the same player can be selected in a number of consecutive periods, the MDP is aperiodic, which implies that the limits in Definition 5.1 are well-defined.

We first show that $\underline{u}(G)$ and $\bar{u}(G)$ coincide in networks where the farsighted player is linked to every myopic opponent. Let $\mathcal{G}_{0}=\left\{G \in \mathcal{G}: \forall i \in I, g_{0 i}=1\right\}$ denote the set of such networks with $n+1$ players. 
Proposition 5.2. Let $\Gamma^{\prime}(G)$ be such that $G \in \mathcal{G}_{0}$. There exists $\bar{\delta}<1$ such that, for every $\delta>\bar{\delta}$,

$$
\underline{u}(G)=\bar{u}(G)=f_{0}(\bar{e}(G))
$$

Proof. See Appendix.

We construct a strategy that guarantees a consumption of $\bar{e}(G)$ units of the good from without any own efforts, for a long-run payoff of $f_{0}(\bar{e}(G))$. If the farsighted player is sufficiently patient, then his SSPE strategy must also achieve at least this long-run payoff. This strategy is analogous to that of the kite example presented in the introduction: (1) establish dependence by exerting an effort of 1 until every myopic player decreases to 0 , and (2) withdrawal of efforts and forcing an increase from the opponents. Steps (1) and (2) are repeated if fewer than $\bar{e}(G)$ players increase after in the withdrawal stage. The complexity of this strategy is rather low, thus the farsighted player does not need the sophistication to compute the SSPE strategies to reach his optimal outcome. Note, however, that this strategy is not necessarily an SSPE itself, as there may be better strategies that guarantee the same outcome, e.g. by reaching the optimum sooner. We will generalize this point to the entire class of networks towards the end of this section.

We now show how $\bar{u}(G)$ is directly related to $\bar{e}(G)$ for a general network $G \in \mathcal{G}$ for arbitrary values of the discount factor.

Proposition 5.3. The upper bound on the farsighted player's expected payoffs in the game $\Gamma^{\prime}(G)$ satisfies

$$
\bar{u}(G)= \begin{cases}f_{0}(\bar{e}(G)) & \text { if } \bar{e}(G)>0, \\ f_{0}(1)-\gamma_{0} & \text { if } \bar{e}(G)=0 .\end{cases}
$$

Proof. Let $M^{\prime}$ be a maximal independent set of $I_{0}$ and $M=M^{\prime} \cap N_{0}(G)$ be a maximum independent set of $N_{0}(G)$, so $\bar{e}(G)=|M|$. For every SSPE $s^{*} \in S^{*}$ it holds that $x^{M^{\prime}} \in A\left(s_{0}^{*}\right)$ by Proposition 3.8. Within the set $X^{\prime}$, the instantaneous payoffs of player 0 are maximized at $x^{M^{\prime}}$, meaning that $\bar{u}(G)=f_{0}(\bar{e}(G))$ if $N_{0} \neq \emptyset$, while $\bar{u}(G)=f_{0}(1)-\gamma_{0}$ if $N_{0}=\emptyset$.

Proposition 5.3 shows that the value of the upper bound is easily calculated as the cardinality of a maximum independent set of the farsighted player's neighborhood and can therefore be computed with only local knowledge of the network.

To provide an expression for $\underline{u}(G)$, we need a few technical definitions first.

Definition 5.4 (Partnerships and closed partnerships). Let $G \in \mathcal{G}$ and $x \in X$. A, possibly empty, union of factions in $x$ is a partnership in $x$. The collection of partnerships in $x$ is denoted by $\mathcal{B}(x)$ and the largest partnership in $x$ by $B(x)$, so $B(x)=\cup_{B \in \mathcal{B}(x)} B$. A partnership $B \in \mathcal{B}(x)$ is closed if $B \subseteq I \backslash N_{0}(G)$ and for every $i \in N_{B}(G)$ it holds that $x\left(\bar{N}_{i}(G) \cap B\right) \geq 1$. The collection of closed partnerships in $x$ is denoted by $\overline{\mathcal{B}}(x)$ and the largest closed partnership in $x$ by $\bar{B}(x)$, so $\bar{B}(x)=\cup_{B \in \overline{\mathcal{B}}(x)} B$. 
Partnerships are unions of factions. It is easily verified that the union of two partnerships is a partnership, which implies that the largest partnership is indeed a partnership. A closed partnership is a partnership whose members provide at least 1 unit of effort for each of their free-riding neighbors. It is easily verified that the union of two closed partnerships is a closed partnership, which implies that the largest closed partnership is indeed a closed partnership. For example, player 2 of the network on the right side of Figure 2 is a closed partnership.

Definition 5.5 (Blocking profile). Let $G \in \mathcal{G}$. The set $X^{\mathrm{b}}$ defined by

$$
X^{\mathrm{b}}=\left\{x^{*} \in X^{*} \mid x_{0}^{*}=1 \text { and } I \backslash N_{0}(G) \subseteq \bar{N}_{\bar{B}\left(x^{*}\right)}(G)\right\}
$$

is the set of blocking profiles.

The set $X^{\mathrm{b}}$ consists of effort profiles such that the farsighted player has an effort level equal to one, all myopic players have an effort level equal to their best response, implying that all players in the neighborhood of the farsighted player have an effort level of 0 and all myopic players have a consumption level greater than or equal to one, and all myopic players outside the neighborhood of the farsighted player derive a consumption level of at least one from the contributions of their neighbors in the largest closed partnership.

The set $X^{\mathrm{b}}$ is always non-empty, since whenever $M$ is a maximal independent set of $I \backslash N_{0}(G)$, then $x^{\{0\} \cup M} \in X^{\mathrm{b}}$. The right side of Figure 2 shows a blocking profile. Further, $X^{*} \backslash X^{\mathrm{b}}$ may be non-empty. For the four-player line network in Figure 5, the given effort profile $x^{*}=(1,0,1 / 2,1 / 2)$ clearly belongs to $X^{*}$, but is not in $X^{\mathrm{b}}$. It is indeed true that $x_{0}^{*}=1$, but the partnership $B=\{2,3\}$ is not a closed partnership, since $x^{*}\left(\bar{N}_{1}(G) \cap B\right)=x_{2}^{*}=1 / 2<1$. It holds that $\bar{B}\left(x^{*}\right)=\emptyset$ and $I \backslash N_{0}(G)=\{2,3\} \not \subseteq=\bar{N}_{\bar{B}\left(x^{*}\right)}(G)$.

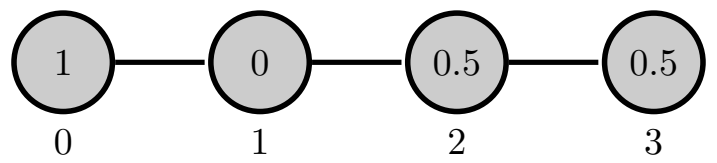

Figure 5: Example showing a static equilibrium profile that is not a blocking profile.

At an effort profile in $X^{\mathrm{b}}$, no player in $I \backslash N_{0}(G)$ reacts to changes in efforts by player 0 as all belong to or are neighbors of a closed partnership. Such a player is "blocked." Some players in $N_{0}(G)$ may also receive contributions greater than or equal to one from their neighbors in the closed partnership. Such players will also not respond to changes in efforts by player 0 and are blocked as well.

For $x \in X^{\mathrm{b}}$, let $I_{0}^{\mathrm{u}}(x)=I_{0} \backslash \bar{N}_{\bar{B}(x)}(G)$ denote the players who are "unblocked," i.e. not blocked by the largest closed partnership. Note that $I_{0}^{\mathrm{u}}(x) \subseteq \bar{N}_{0}(G)$ as all other players are blocked by a closed partnership by Definition 5.5, but in general $I_{0}^{\mathrm{u}}(x)$ is not equal to $\bar{N}_{0}(G)$.

For every $G \in \mathcal{G}$, we define $\underline{e}(G)$ by

$$
\underline{e}(G)=\min _{x \in X^{\mathrm{b}}} \bar{e}\left(\left.G\right|_{I_{0}^{\mathrm{u}}(x)}\right)
$$


where for a given network $G$ and a given subset of the players $J \subseteq I_{0},\left.G\right|_{J}$ denotes the network resulting from removing all players in $I_{0} \backslash J$ and the links related to these players from the network $G$.

The value $\underline{e}(G)$ equals the minimum number of players that the farsighted player can exploit no matter what the initial state is. The reason for this is the following. Since in a blocking effort profile $x \in X^{\mathrm{b}}$ all players outside of $I_{0}^{\mathrm{u}}(x)$ are getting more than one unit of effort from some members of a closed partnership, we consider the subgraph of $G$ where these players are removed. From such a subgraph we take the cardinality of the maximum independent set $\bar{e}\left(\left.G\right|_{I_{0}^{\mathrm{u}}(x)}\right)$, since, as seen previously in Proposition 5.3, getting one unit of effort from the maximum independent set of his neighborhood is the best instantaneous payoff the farsighted player can hope for. In addition, this payoff is attainable, as we will show in the next proposition. By taking the minimum over effort profiles in $X^{\mathrm{b}}$, the value $\underline{e}(G)$ gives the exact number of units of the public good that is attainable for even the worst choice of the starting state.

Proposition 5.6. There exists $\bar{\delta}<1$ such that, for every $\delta>\bar{\delta}$, the lower bound on the farsighted player's expected payoffs in the game $\Gamma^{\prime}(G)$ is given by

$$
\underline{u}(G)= \begin{cases}f_{0}(\underline{e}(G)) & \text { if } \underline{e}(G)>0, \\ f_{0}(1)-\gamma_{0} & \text { if } \underline{e}(G)=0 .\end{cases}
$$

Proof. See Appendix.

By Proposition 5.6, the value of the lower bound hinges on the maximum number of independent neighbors of 0 that cannot be blocked. Every such player is liable to exploitation by the farsighted player via the dependence-withdrawal strategy.

Similarly to Proposition 5.2 , there are relatively simple strategies to guarantee the long-run payoff of the lower bound. The farsighted player first makes sure that a blocking effort profile is reached. This involves exerting an effort of 1 as long as there are players in $N_{0}(G)$ with positive efforts and contributing 0 if all players in $N_{0}(G)$ are free riding. It can be shown that such a strategy leads to a blocking profile with probability one. Next the farsighted player exploits a maximum independent set of unblocked players.

The characterizations of the bounds leads us to our main comparative statics results. For a network $G$ with $g_{i j}=0$, let $G_{i j}$ be the network we get by adding the link $\{i, j\}$ to $G$.

Theorem 5.7. Let $G \in \mathcal{G}$ and $i, j \in I_{0}$ be such that $g_{i j}=0$.

1. (Sociability) If $0 \in\{i, j\}$, then $\bar{u}(G) \leq \bar{u}\left(G_{i j}\right)$ and $\underline{u}(G) \leq \underline{u}\left(G_{i j}\right)$. In addition, if $N_{i}(G) \cap N_{j}(G)=$ $\emptyset$ we have $\bar{u}(G)<\bar{u}\left(G_{i j}\right)$.

2. (Jealousy) If $i, j \in N_{0}(G)$, then $\bar{u}(G) \geq \bar{u}\left(G_{i j}\right)$ and $\underline{u}(G) \geq \underline{u}\left(G_{i j}\right)$.

3. Otherwise, $\bar{u}(G)=\bar{u}\left(G_{i j}\right)$. 
Proof. By Propositions 5.3 and 5.6, and by the strict monotonicity of $f_{0}$, it is sufficient to show the analogue of Theorem 5.7 after replacing $\bar{u}$ with $\bar{e}$ and $\underline{u}$ with $\underline{e}$ in all three statements. The substitution also makes use of $f_{0}(1)>f_{0}(1)-\gamma_{0}$ for the cases $\underline{e}(G)=0$ and $\bar{e}(G)=0$. Let $X_{i j}^{\mathrm{b}}$ be the set of blocking profiles for the network $G_{i j}$.

1. Without loss of generality, let $i=0$. Let $K^{\prime} \in \mathcal{K}_{0}(G)$. Since $g_{i j}=0$, it holds that $j \notin K^{\prime}$. After adding the link between $i$ and $j$, the set $K^{\prime}$ is therefore still independent, so $K^{\prime} \in \mathcal{K}_{0}\left(G_{i j}\right)$. It follows that

$$
\bar{e}(G)=\max _{K \in \mathcal{K}_{0}(G)}|K| \leq \max _{K \in \mathcal{K}_{0}\left(G_{i j}\right)}|K|=\bar{e}\left(G_{i j}\right) .
$$

In case $N_{0}(G) \cap N_{j}(G)=\emptyset$, it holds that $K^{\prime} \cup\{j\} \in \mathcal{K}_{0}\left(G_{i j}\right)$, so the weak inequality above becomes strict and $\bar{e}(G)<\bar{e}\left(G_{i j}\right)$.

Let $x \in X_{i j}^{\mathrm{b}}$ and let $B$ be the related largest closed partnership in $G_{i j}$. Since $j \in N_{0}\left(G_{i j}\right)$, it holds that $j \notin B$. If $j \notin N_{B}\left(G_{i j}\right)$, then define $x^{*} \in X$ by $x_{j}^{*}=1$ and $x_{-j}^{*}=x_{-j}$. It holds that $x^{*} \in X^{\mathrm{b}}$ and $\bar{B}\left(x^{*}\right)=B \cup\{j\}$. Player $j$ was unblocked in $G_{i j}$ and is part of $\bar{B}\left(x^{*}\right)$ in $G$. It follows that $\bar{e}\left(\left.G\right|_{I_{0} \backslash \bar{N}_{B \cup\{j\}}(G)}\right) \leq \bar{e}\left(\left.G_{i j}\right|_{I_{0} \backslash \bar{N}_{B}\left(G_{i j}\right)}\right)$. If $j \in N_{B}\left(G_{i j}\right)$, then since $B$ is a closed partnership in $G_{i j}$, a contribution of 0 is a best-response for player $j$ against $x$ in the network $G$, so $x \in X^{\mathrm{b}}$ and $\bar{B}(x)=B$. We have that $\bar{e}\left(\left.G\right|_{I_{0} \backslash \bar{N}_{B}(G)}\right)=\bar{e}\left(\left.G_{i j}\right|_{I_{0} \backslash \bar{N}_{B}\left(G_{i j}\right)}\right)$. We conclude that $\underline{e}(G) \leq \underline{e}\left(G_{i j}\right)$.

2. Let $i, j \in N_{0}(G)$. Let $K^{\prime} \in \mathcal{K}_{0}\left(G_{i j}\right)$. After deleting the link between $i$ and $j$, the set $K^{\prime}$ is still independent, so $K^{\prime} \in \mathcal{K}_{0}(G)$. It follows that

$$
\bar{e}\left(G_{i j}\right)=\max _{K \in \mathcal{K}_{0}\left(G_{i j}\right)}|K| \leq \max _{K \in \mathcal{K}_{0}(G)}|K|=\bar{e}(G) .
$$

We first observe that $X^{\mathrm{b}}=X_{i j}^{\mathrm{b}}$. Let $J$ be any subset of $\bar{N}_{0}(G)$. Since any independent set of $\left.G_{i j}\right|_{J}$ is also an independent set of $\left.G\right|_{J}$, it follows that $\underline{e}(G) \geq \underline{e}\left(G_{i j}\right)$.

3. In this case $\left.G\right|_{\bar{N}_{0}(G)}=\left.G_{i j}\right|_{N_{0}\left(G_{i j}\right)}$, which leads to the desired conclusion.

A new link is either beneficial or neutral to the farsighted player if it adds a myopic player to his neighborhood. This result is called Sociability, as the farsighted player always weakly benefits from forming new links. A new link is harmful or neutral to the farsighted player if two of his unlinked neighbors become linked. This result is called Jealousy, as the farsighted player is always weakly harmed by his neighbors linking up with each other. Adding any other links has no effect on the upper bound, while their effect on the lower bound is ambiguous. This fact will be demonstrated in Examples 5.8 and 5.9 ,

The comparison results concerning the upper bound are achieved as straightforward consequences of its characterization as the cardinality of a maximum independent set of the farsighted player's neighborhood. Adding a player into the neighborhood increases the number of players whose efforts are enjoyed by the farsighted player, although depending on the connections the upper bound may not change. In case the new neighbor shares no connection with any of the old ones, the upper bound is 
guaranteed to increase, implying that the farsighted player would prefer linking to more distant players in the network rather than to neighbors of his neighbors.

Changes in the network affecting the neighborhood of the farsighted player have the same effect on the lower bound. A new link between two neighbors of the farsighted player has no effect on the set of blocking profiles but it can decrease the cardinality of the maximum independent sets of the remaining players, lowering the lower bound. Adding a new neighbor reduces set of blocking profiles while potentially increasing the cardinality of maximum independent sets, both effects work to increase the lower bound. In all other cases the new link changes the set of blocking profiles in a way that can lead to an increase or to a decrease in the lower bound. This is demonstrated by the following two examples which make up the remainder of this section.

Example 5.8. Consider a network of five players $\{0,1,2,3,4\}$ with the following four links: $\{0,1\}$, $\{1,2\},\{0,3\}$ and $\{3,4\}$. In this network, the value of the lower bound is $f_{0}(1)-\gamma_{0}$, since both neighbors of the farsighted player can be blocked. The left side of Figure 6 shows the network and an appropriate blocking profile.
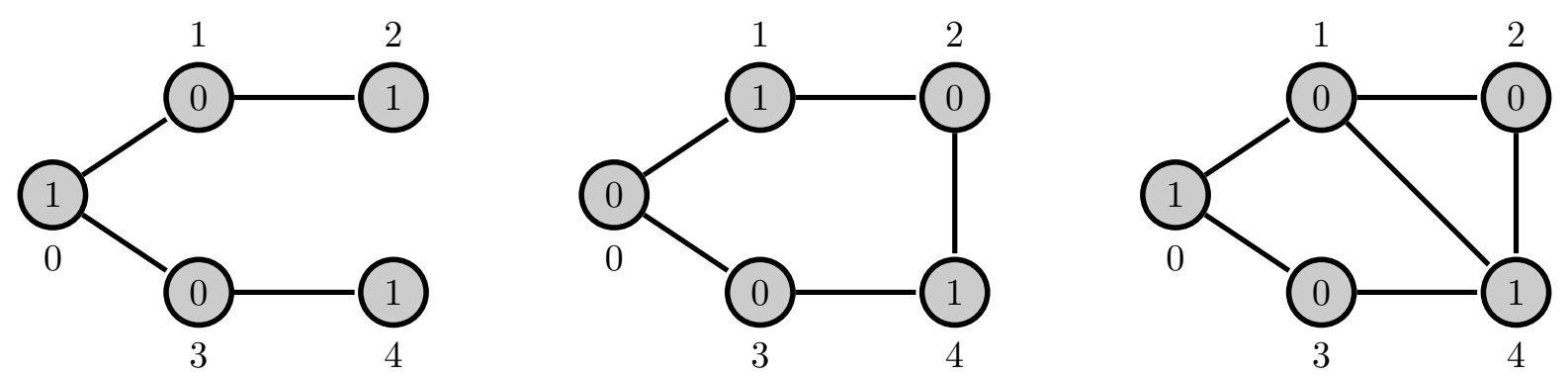

Figure 6: The network and worst blocking effort profile of Example 5.8 initially (left), adding the link $\{2,4\}$ (middle), and adding the link $\{1,4\}$ (right). After linking the two non-neighbors of player 0 , his two neighbors cannot be blocked at the same time. However, adding another link they can be blocked again.

Adding the link $\{2,4\}$ eliminates this blocking effort profile as players 2 and 4 both exerting an effort of 1 is no longer stable for myopic deviation. Hence, at least one of players 1 and 3 will always be free to provide for player 0 , and the value of the lower bound increases to $f_{0}(1)$. The resulting network with a new blocking profile is shown in the middle of Figure 6. Now consider adding the link $\{1,4\}$ to the new network. Then, the profile in which player 4 has an effort of 1 and all other myopic players are free riding once again blocks both neighbors of the farsighted player. This resulting network is shown on the right side of the figure.

This illustrates that adding a link between two non-neighbors can benefit the farsighted player, while adding a link between a neighbor and a non-neighbor may harm him.

Example 5.8 shows two simple cases through which the lower bound can be influenced. Linking the farsighted player's neighbors to non-neighbors can make it easier to achieve blocking effort profiles due to more links, while linking two non-neighbors to each other can make it harder, as closed partnerships will be harder to find. 
While the above reasoning holds for many networks, there are examples where linking a neighbor to a non-neighbor, or deleting a link between two non-neighbors both have the potential to increase the lower bound if the original blocking profile is sensitive to any changes in the network. This is shown in the following example.

Example 5.9. Consider a network with 17 players. The farsighted player is linked to ten myopic players, none of which have links between them. The remaining six myopic players are grouped into two cliques of three players, but there are no links between the cliques. One of the farsighted player's neighbors has no other neighbors. The other nine neighbors are connected to three other players each: one player from one clique and two players from the other. No two of these nine players have the same set of neighbors.

In this network, the profile where the six clique members are each exerting an effort of $1 / 3$ each blocks the nine neighbors, and this is the only blocking profile that will block all of them, while the tenth neighbor cannot be blocked in any way, hence the value of the lower bound is $f_{0}(1)$. Figure 7 shows this network and blocking profile.

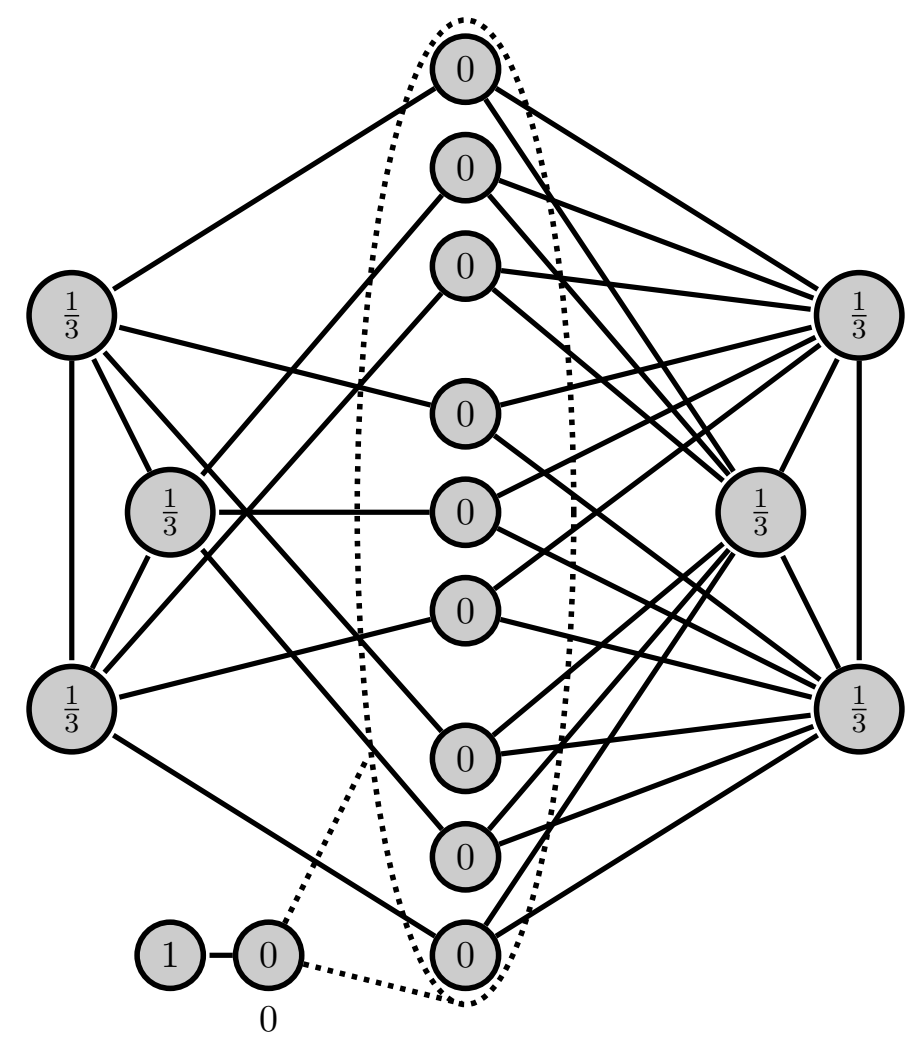

Figure 7: The network and the worst blocking profile of Example 5.9 The dotted ellipsis and lines indicate the neighborhood of the farsighted player.

Suppose that we remove a link in the cliques with which each of the nine neighbors has one link so that this clique now forms a line of three players. As a result, the blocking profile in Figure 7 is no longer admissible, since they are no longer in a partnership. The new worst blocking profile is the one in which the center player of the line is a free rider and the other two players have an effort of 1 , 
while the other clique has two free riders and one player with 1 effort. The value of the lower bound increases to $f_{0}(2)$. The new blocking profile is shown on the left side of Figure 8 .

Finally, suppose that we take the original network and add a link between the tenth neighbor and one of the members of the clique with which the nine neighbors have one link. Then, the original blocking profile of Figure 7 will again be inadmissible as it provides an effort of only $1 / 3$ to the tenth neighbor, thus it is not a blocking profile. The worst blocking profile is the one where each clique has a single player with 1 effort, including the one to whom the tenth neighbor is now linked. In this profile the farsighted player is no longer able to exploit the tenth player, but instead gains from two of his nine other neighbors, who are now linked to trios of free riders. The value of the lower bound again increases to $f_{0}(2)$. This profile is shown on the right side of Figure 6 .
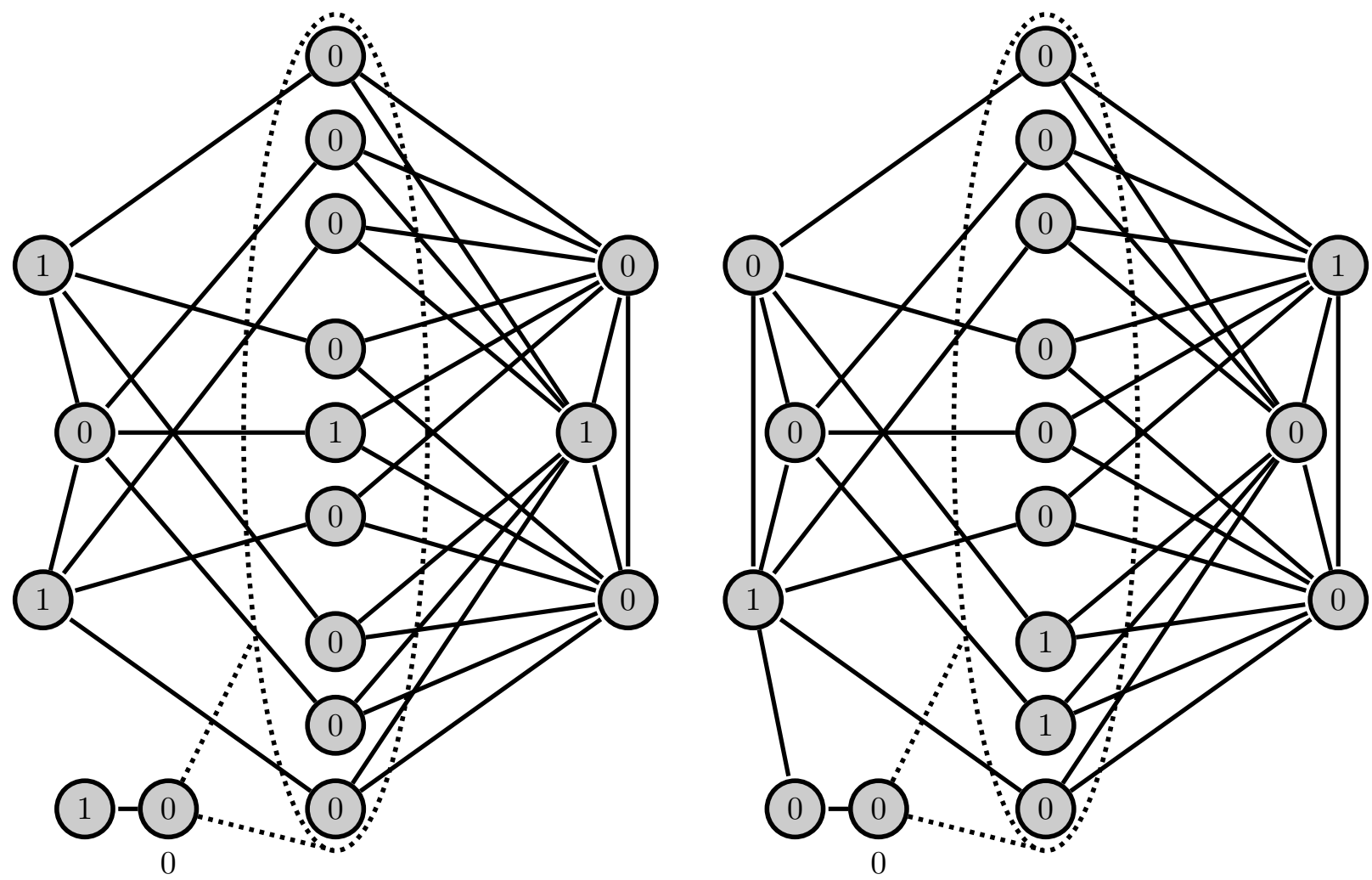

Figure 8: The network and a worst effort profile of Example 5.9 with one link from one of the cliques removed (left) and one link added (right). The number of neighbors that can be exploited increases to 2 in both cases.

As a consequence of Theorem 5.7, for a fixed number of players, the star network with the farsighted player in the center maximizes both the lower and the upper bound, and they are equal to $f_{0}(n-1)$ by Proposition 5.2. If linking to new players is a costless option, then the first statement of Theorem 5.7 guarantees that the farsighted player will link to all myopic players. Additionally, if the farsighted player can sever links running between myopic players, then the second statement implies that the star network will be reached by severing all links between his neighbors. However, as shown by Example 5.8, deleting a link between two myopic players who are not both neighbors of the farsighted player may 
decrease the lower bound. Furthermore, Example 5.9 shows that adding a link between two myopic players can increase the value of the lower bound. These two facts provide stumbling blocks for a farsighted player from reaching the star network.

\section{Conclusion}

In this paper we consider the private provision of local public goods game, a gameclass with a wide array of applicability and a strong body of theoretical contributions. We build a model of manipulation and exploitation of a myopic population by a single farsighted player with the goal of analyzing exploitative behavior in various social networks.

We consider a dynamic and stochastic setting such that in every time period a randomly selected player is given a chance to revise his exerted effort into the local public good. With this dynamic, a game played by a myopic population is known to converge to a Nash effort profile. The farsighted player's optimal strategies correspond to optimal policies of an MDP, and hence, stationary subgame perfect equilibria of this game exist for every network structure and discount factor of the farsighted player. We find that every network and every SSPE permits the existence of absorbing effort profiles that are stable for deviations given the strategies of the players and that the set of absorbing profiles is neither a subset nor a superset of the set of Nash equilibria of the static game.

Unless stable partnerships of myopic players are able to form, players with lower consumption will increase their efforts, while players with high consumption will lower theirs. As these two effects are mutually reinforcing one another, we observe a tendency towards a rising inequality of production of the local public good within the myopic population. Consequently, partnerships may form with clusters of producers being surrounded by free riders.

In the long run, every member of a partnership of myopic players outside the farsighted player's neighborhood will produce a constant effort level, as the free riders surrounding the partnerships will dampen the effects of any future changes in efforts by the farsighted player. This means that players who do not share a neighbor with the farsighted player will also produce constant effort levels in the long run. As a result, the impact of the farsighted player on the myopic population are both short-lived and localized within the network.

Finally, we compare networks based on the opportunities of exploitation they provide to the farsighted player. To make the comparison, we analyze the farsighted player's minimum and maximum available long-run payoff they can attain in a network by playing optimally. We provide characterizations for both bounds by the use of maximum independent sets of (some subset of) the farsighted player's neighborhood, and show that the value of the lower bound is reachable from any starting state by a very simple dependence-withdrawal strategy. If the farsighted player is linked to every myopic opponent, the upper bound is also reachable with this strategy. The farsighted player is Sociable, as both bounds increase from the addition of a new link between him and a myopic player, and Jealous, as both bounds decrease from the addition of a new link between two of his neighbors. From this 
we conclude that the star network maximizes the farsighted player's prospects for exploitation, but there are cases when adding a link between two myopic players increases his minimum and maximum evaluation.

This paper's results has interesting implications on the case of multiple farsighted players as well. Equilibrium existence in this case no longer follows from the theory of MDPs. On the other hand, since the farsighted player's impact on the myopic population is localized, we conjecture that multiple farsighted players with sufficient distance from each other will not interact with one another in the long run, and most dynamic results of this paper are upheld. Our results therefore can be generalized to multiple farsighted players provided that they appear sparsely in the network. An explicit analysis of this case is left for future research.

\section{A Appendix: Proofs}

Before proving Proposition 4.7, we introduce a number of technical concepts and results. For $x \in X$, $j \in I$, and $J \subseteq I$ we use the notation $N_{j}^{+}(x)=N_{j} \cap I^{+}(x)$ and $N_{J}^{+}(x)=N_{J} \cap I^{+}(x)$ for the neighbors of $j$, respectively $J$, with positive contributions. Next, we introduce the notion of a buffer set, which corresponds to all the neighbors whose contributions a given player can usurp by making repeated use of Proposition 4.4 .

Definition A.1 (Buffer set). Let $x^{0} \in X$ and $j \in I$ be given such that $x_{j}^{0}>0$. Define

$$
D_{j}^{0}\left(x^{0}\right)=\left\{i \in N_{j}^{+}\left(x^{0}\right): c_{i}\left(x^{0}\right)>c_{j}\left(x^{0}\right)\right\} .
$$

For $k \geq 1$, define $x^{k} \in X$ and $D_{j}^{k}\left(x^{0}\right) \subseteq N_{j}$ recursively by:

$$
\begin{aligned}
x_{i}^{k} & = \begin{cases}0 & \text { if } i \in D_{j}^{k-1}\left(x^{0}\right), \\
\max \left\{0,1-x^{k-1}\left(N_{j} \backslash D_{j}^{k-1}\left(x^{0}\right)\right)\right\} & \text { if } i=j, \\
x_{i}^{k-1} & \text { otherwise. }\end{cases} \\
D_{j}^{k}\left(x^{0}\right) & =D_{j}^{k-1}\left(x^{0}\right) \cup\left\{i \in N_{j}^{+}\left(x^{k}\right): c_{i}\left(x^{k}\right)>c_{j}\left(x^{k}\right)\right\}=D_{j}^{k-1}\left(x^{0}\right) \cup D_{j}^{0}\left(x^{k}\right) .
\end{aligned}
$$

The set $D_{j}\left(x^{0}\right)=\cup_{k \in \mathbb{N}} D_{j}^{k}\left(x^{0}\right)$ is the buffer set of player $j$ in effort profile $x^{0}$.

Since the set $N_{j}$ is finite, there is $k^{\prime} \in \mathbb{N}$ such that $D_{j}^{k^{\prime}}\left(x_{0}\right)=D_{j}^{k^{\prime}-1}\left(x_{0}\right)$, so $D_{j}^{k^{\prime}}\left(x^{0}\right)=D_{j}\left(x^{0}\right)$. The buffer set $D_{j}\left(x^{0}\right)$ consists of all the players whose efforts can be usurped by player $j$ starting from effort profile $x^{0}$ according to a repeated use of Proposition 4.4

For $k \geq 0$, the effort profile $x^{k+1}$ with $x_{j}^{k+1}>0$ results after the efforts of the players in $D_{j}^{k}\left(x^{0}\right)$ have been usurped. Proposition 4.4 guarantees that $x_{j}^{k+1} \geq x_{j}^{k}>0$. Additionally, for every $i \in D_{j}\left(x^{0}\right)$ we have $c_{i}\left(x^{k^{\prime}}\right) \geq c_{j}\left(x^{k^{\prime}}\right)$. By Proposition 4.4 for every $s \in S_{0} \times\left\{b_{I}\right\}$, we have $x^{k+1} \in R_{s}^{D_{j}^{k}\left(x^{0}\right) \cup\{j\}}\left(x^{0}\right)$. It follows that $x^{k^{\prime}} \in R_{s}^{D_{j}\left(x^{0}\right) \cup\{j\}}\left(x^{0}\right)$.

Definition A.2 (Valley). A non-empty set $V \subseteq I^{+}(x)$ is a valley at $x \in X$ if $V$ is connected, for every $j, j^{\prime} \in V, c_{j}(x)=c_{j^{\prime}}(x)$, and for every $i \in N_{V}^{+}(x), c_{i}(x)>c_{j}(x)$. 
Every valley is a subset of an island, since all players in a valley are connected and have positive effort levels. Also, every island contains at least one valley. Every faction is a valley, since all players in a faction have consumption equal to 1 and there are no players with positive effort levels in the neighborhood of a faction.

Lemma A.3. Let $x^{0} \in X$ and a valley $V \subseteq I^{+}\left(x^{0}\right)$ be given. For every $k \geq 0$, for every $j \in V$, and for every $i \in N_{j}^{+}\left(x^{0}\right) \backslash D_{j}^{k}\left(x^{0}\right)$ it holds that

$$
i \in D_{j}^{k+1}\left(x^{0}\right) \backslash D_{j}^{k}\left(x^{0}\right) \Longleftrightarrow D_{j}^{k}\left(x^{0}\right) \nsubseteq N_{i} .
$$

Proof. We start by proving the equivalence in 2 for $k=0$. Let some $j \in V$ and some $i \in N_{j}^{+}\left(x^{0}\right) \backslash$ $D_{j}^{0}\left(x^{0}\right)$ be given. Observe that $c_{i}\left(x^{0}\right)=c_{j}\left(x^{0}\right)$ since $V$ is a valley and $i \notin D_{j}^{0}\left(x^{0}\right)$.

$(\Rightarrow)$ From $i \in D_{j}^{1}\left(x^{0}\right)$, it follows that $c_{i}\left(x^{1}\right)>c_{j}\left(x^{1}\right)$. By Definition A.1, we have that

$$
c_{j}\left(x^{1}\right)-c_{j}\left(x^{0}\right)=x_{j}^{1}-x_{j}^{0}-x^{0}\left(D_{j}^{0}\left(x^{0}\right)\right) .
$$

Suppose $D_{j}^{0}\left(x^{0}\right) \subseteq N_{i}$. By $i \in N_{j}$, we have

$$
c_{i}\left(x^{1}\right)-c_{i}\left(x^{0}\right)=x_{j}^{1}-x_{j}^{0}-x^{0}\left(D_{j}^{0}\left(x^{0}\right)\right),
$$

so $c_{i}\left(x^{0}\right)=c_{j}\left(x^{0}\right)$ implies $c_{i}\left(x^{1}\right)=c_{j}\left(x^{1}\right)$, a contradiction to $c_{i}\left(x^{1}\right)>c_{j}\left(x^{1}\right)$. Consequently, it holds that $D_{j}^{0}\left(x^{0}\right) \nsubseteq N_{i}$.

$(\Leftarrow)$ From $D_{j}^{0}\left(x^{0}\right) \nsubseteq N_{i}$ it follows that

$$
c_{i}\left(x^{1}\right)-c_{i}\left(x^{0}\right)=x_{j}^{1}-x_{j}^{0}-x^{0}\left(D_{j}^{0}\left(x^{0}\right) \cap N_{i}\right)>x_{j}^{1}-x_{j}^{0}-x^{0}\left(D_{j}^{0}\left(x^{0}\right)\right),
$$

so $c_{i}\left(x^{0}\right)=c_{j}\left(x^{0}\right)$ implies $c_{i}\left(x^{1}\right)>c_{j}\left(x^{1}\right)$, hence $i \in D_{j}^{1}\left(x^{0}\right)$.

Assume, for some $k \geq 1$, for every $j \in V$, and, for every $i \in N_{j}^{+}\left(x^{0}\right) \backslash D_{j}^{k-1}\left(x^{0}\right)$, we have $c_{i}\left(x^{k-1}\right)=$ $c_{j}\left(x^{k-1}\right)$ as well as the equivalence $i \in D_{j}^{k}\left(x^{0}\right) \backslash D_{j}^{k-1}\left(x^{0}\right) \Leftrightarrow D_{j}^{k-1}\left(x^{0}\right) \nsubseteq N_{i}$. We now show that the same assertion holds for $k+1$.

Let some $j \in V$ and some $i \in N_{j}^{+}\left(x^{0}\right) \backslash D_{j}^{k}\left(x^{0}\right)$ be given. By Definition A.1, we have that

$$
c_{j}\left(x^{k}\right)-c_{j}\left(x^{k-1}\right)=x_{j}^{k}-x_{j}^{k-1}-x^{k-1}\left(D_{j}^{k-1}\left(x^{0}\right)\right) .
$$

Since $i \notin D_{j}^{k}\left(x^{0}\right)$, it holds that $D_{j}^{k-1}\left(x^{0}\right) \subseteq N_{i}$, so

$$
c_{i}\left(x^{k}\right)-c_{i}\left(x^{k-1}\right)=x_{j}^{k}-x_{j}^{k-1}-x^{k-1}\left(D_{j}^{k-1}\left(x^{0}\right)\right),
$$

so $c_{i}\left(x^{k-1}\right)=c_{j}\left(x^{k-1}\right)$ implies $c_{i}\left(x^{k}\right)=c_{j}\left(x^{k}\right)$.

$(\Rightarrow)$ From $i \in D_{j}^{k+1}\left(x^{0}\right)$, it follows that $c_{i}\left(x^{k+1}\right)>c_{j}\left(x^{k+1}\right)$. By Definition A.1, we have that

$$
c_{j}\left(x^{k+1}\right)-c_{j}\left(x^{k}\right)=x_{j}^{k+1}-x_{j}^{k}-x^{k}\left(D_{j}^{k}\left(x^{0}\right)\right) .
$$

Suppose $D_{j}^{k}\left(x^{0}\right) \subseteq N_{i}$. By $i \in N_{j}$, we have

$$
c_{i}\left(x^{k+1}\right)-c_{i}\left(x^{k}\right)=x_{j}^{k+1}-x_{j}^{k}-x^{k}\left(D_{j}^{k}\left(x^{0}\right)\right),
$$


so $c_{i}\left(x^{k}\right)=c_{j}\left(x^{k}\right)$ implies $c_{i}\left(x^{k+1}\right)=c_{j}\left(x^{k+1}\right)$, a contradiction to $c_{i}\left(x^{k+1}\right)>c_{j}\left(x^{k+1}\right)$. Consequently, it holds that $D_{j}^{k}\left(x^{0}\right) \nsubseteq N_{i}$.

$(\Leftarrow)$ From $D_{j}^{k}\left(x^{0}\right) \nsubseteq N_{i}$ it follows that

$$
c_{i}\left(x^{k+1}\right)-c_{i}\left(x^{k}\right)=x_{j}^{k+1}-x_{j}^{k}-x^{k}\left(D_{j}^{k}\left(x^{0}\right) \cap N_{i}\right)>x_{j}^{k+1}-x_{j}^{k}-x^{k}\left(D_{j}^{k}\left(x^{0}\right)\right),
$$

so $c_{i}\left(x^{k}\right)=c_{j}\left(x^{k}\right)$ implies $c_{i}\left(x^{k+1}\right)>c_{j}\left(x^{k+1}\right)$, hence $i \in D_{j}^{k+1}\left(x^{0}\right)$.

Lemma A.4. Let $x \in X$ and a valley $V \subseteq I^{+}(x)$ be given. Let $i, j \in V$ be such that $g_{i j}=1$.

1. If $j \notin D_{i}(x)$ and $i \notin D_{j}(x)$, then $D_{i}(x)=D_{j}(x)$.

2. If $j \notin D_{i}(x)$ and $i \in D_{j}(x)$, then $D_{i}(x) \subset D_{j}(x)$.

Proof. It is sufficient to show that $i \notin D_{j}(x)$ implies $D_{j}(x) \subseteq D_{i}(x)$. Let $i \notin D_{j}(x)$, so by Lemma A.3. for every $k \geq 0, D_{j}^{k}(x) \subseteq N_{i}$. We show that for every $k \geq 0, D_{j}^{k}(x) \subseteq D_{i}^{k}(x)$.

For every $i^{\prime} \in D_{j}^{0}(x)$ we have $c_{i}(x)=c_{j}(x)<c_{i^{\prime}}(x)$, so since $D_{j}^{0}(x) \subseteq N_{i}$, we have $i^{\prime} \in D_{i}^{0}(x)$, so $D_{j}^{0}(x) \subseteq D_{i}^{0}(x)$.

We complete the proof by showing that, for every $k \geq 0, D_{j}^{k}(x) \subseteq D_{i}^{k}(x)$ implies $D_{j}^{k+1}(x) \subseteq$ $D_{i}^{k+1}(x)$. Suppose $D_{j}^{k}(x) \subseteq D_{i}^{k}(x)$ but $D_{j}^{k+1}(x) \nsubseteq D_{i}^{k+1}(x)$. Then there exists $i^{\prime} \in D_{j}^{k+1}(x)$ such that $i^{\prime} \notin D_{i}^{k+1}(x)$. It follows that $i^{\prime} \notin D_{i}^{k}(x)$, so $i^{\prime} \notin D_{j}^{k}(x)$. We have $i^{\prime} \notin D_{j}^{0}(x)$, hence it holds $c_{i^{\prime}}(x)=c_{j}(x)$. It follows that $i^{\prime} \in V$. Since $D_{j}^{k+1}(x) \subseteq N_{i}$, we have $i^{\prime} \in N_{i}$. By Lemma A.3. $i^{\prime} \in D_{j}^{k+1}(x)$ implies $D_{j}^{k}(x) \nsubseteq N_{i^{\prime}}$, while $i^{\prime} \notin D_{i}^{k+1}(x)$ implies $D_{i}^{k}(x) \subseteq N_{i^{\prime}}$, contradicting $D_{j}^{k}(x) \subseteq D_{i}^{k}(x)$.

The next lemma provides conditions on $x \in X$ such that for every player $j^{\prime}$ in a given valley at $x$ there exists a connected subset of the valley such that every player in the subset can usurp all the contributing neighbors of the subset as well as $D_{j^{\prime}}(x)$ thereby creating a buffer around the subset.

Lemma A.5. Let $s \in S_{0} \times\left\{b_{I}\right\}$. Let $x \in X$ be a recurrent effort profile and let $V \subseteq I^{+}(x) \backslash N_{0}$ be a valley at $x$. For every $j^{\prime} \in V$ there exists $\emptyset \neq L \subseteq V, D=N_{L}^{+}(x)$, and $y \in R_{s}^{L \cup D}(x)$ such that $D_{j^{\prime}}(x) \subseteq D$ and:

1. For every $j \in L, D_{j}(x)=D$.

2. $L$ is an island at $y$.

Proof. Let some $j^{\prime} \in V$ be given. The following algorithm ends with a connected $L \subseteq V$ such that for every $j \in L, D_{j}(x)=N_{L}^{+}(x) \supseteq D_{j^{\prime}}(x)$ :

Step 0. Define $k=0, L^{k}=\left\{j^{\prime}\right\}$, and $D^{k}=D_{j^{\prime}}(x)$.

Increase $k$ by 1 and go to Step 1 .

Step 1. If $D^{k}=N_{L^{k}}^{+}(x)$, then terminate. Otherwise, go to Step 2 .

Step 2. Take $i^{k} \in N_{L^{k}}^{+}(x) \backslash D^{k}$ and $j^{k} \in L^{k} \cap N_{i^{k}}$. If $j^{k} \notin D_{i^{k}}(x)$, then define $L^{k+1}=L^{k} \cup\left\{i^{k}\right\}$ and $D^{k+1}=D^{k}$. 
If $j^{k} \in D_{i^{k}}(x)$, then define $L^{k+1}=\left\{i^{k}\right\}$ and $D^{k+1}=D_{i^{k}}(x)$.

Increase $k$ by 1 and go to Step 1 .

It holds that $L^{0}=\left\{j^{\prime}\right\}$, so $L^{0} \subseteq V$ and, for every $j \in L^{0}, D_{j}(x)=D^{0}$. Assume, for some $k \geq 0$, $L^{k} \subseteq V$ and, for every $j \in L^{k}, D_{j}(x)=D^{k}$. We show that $L^{k+1} \subseteq V$ and, for every $j \in L^{k+1}$, $D_{j}(x)=D^{k+1}$. Since $i^{k} \in N_{L^{k}}^{+}(x) \backslash D_{j^{k}}(x)$, it holds by Definition A.1 and the definition of a valley that $c_{i^{k}}(x)=c_{j^{k}}(x)$, so $i^{k} \in V$. It follows that $L^{k+1} \subseteq V$. If $j^{k} \notin D_{i^{k}}(x)$, then the first part of Lemma A.4 implies $D_{i^{k}}(x)=D_{j^{k}}(x)$, so together with the assumption that, for every $j \in L^{k}, D_{j}(x)=D^{k}$, we find that for every $j \in L^{k+1}, D_{j}(x)=D^{k}=D^{k+1}$. If $j^{k} \in D_{i^{k}}(x)$, then $L^{k+1}=\left\{i^{k}\right\}$ and $D^{k+1}=D_{i^{k}}(x)$, so obviously, for every $j \in L^{k+1}, D_{j}(x)=D^{k+1}$.

We show next that the algorithm terminates in a finite number of steps. If $j^{k} \in D_{i^{k}}(x)$, then it follows by the second part of Lemma A.4 that $D_{j^{k}}(x) \subset D_{i^{k}}(x)$, so $D^{k} \subset D^{k+1}$. If $j^{k} \notin D_{i^{k}}(x)$, then $D^{k}=D^{k+1}$ and $L^{k} \subset L^{k+1}$. The set $D^{k}$ is therefore monotonically increasing. In iterations where it is constant, the set $L^{k}$ is strictly increasing. Since there are only finitely many agents, the algorithm must terminate in Step 1 after a finite number of steps, say $\ell$. We define $L=L^{\ell}$ and $D=D^{\ell}$. It follows easily that $L$ is connected and, for every $j \in L, D_{j}(x)=N_{L}^{+}(x) \supseteq D_{j^{\prime}}(x)$.

Take a player $j \in L$ and define $y=x^{k^{\prime}} \in R_{s}^{\{j\} \cup D}\left(x^{0}\right)$, where $x^{k^{\prime}}$ with $x_{j}^{k^{\prime}}>0$ is the effort profile that results after player $j$ usurps the players in $D$, see below Definition A.1. For every $i \in D, y_{i}=0$. Since $D=N_{L}^{+}(x)$ and $y(D)=0, L$ is an island in $y$.

Proposition 4.7 (Factions are either completely inside or completely outside $N_{0}$ ). Let $s \in S_{0} \times\left\{b_{I}\right\}$ and let $x \in X$ be a recurrent profile in $s$. If $\left|[x]_{s}\right|>1$, then there exists $y \in[x]_{s} \cap X^{*}$ such that for every $F \in \mathcal{F}(y)$ it holds that either $F \subseteq N_{0}$ or $F \cap N_{0}=\emptyset$.

Proof. By Proposition 4.3 there exists $x^{1} \in R_{s}^{I}(x)$ such that $x_{0}^{1}=x_{0}$ and $x^{1} \in X^{*}$. If no faction in $x^{1}$ has members in both $N_{0}$ and $I \backslash N_{0}$, then we are done, so consider the case where there exists a faction $F \in \mathcal{F}\left(x^{1}\right)$ such that $F \cap N_{0} \neq \emptyset$ and $F \backslash N_{0} \neq \emptyset$.

Since $\left|[x]_{s}\right|>1$, we must have that either $s_{0}\left(x^{1}\right)>x_{0}^{1}$ or $s_{0}\left(x^{1}\right)<x_{0}^{1}$. Suppose $s_{0}\left(x^{1}\right)>x_{0}^{1}$. Let $x^{2}$ be the effort profile that results from $x^{1}$ after a revision by player 0 . For every $i \in F \cap N_{0}$, for every $j \in F \backslash N_{0}$, we have $c_{i}\left(x_{i}^{2}\right)>c_{j}\left(x_{j}^{2}\right)=1$. Let $V$ be a component of $F \backslash N_{0}$. It is easily verified that $V$ is a valley. By Lemma A.5 there exists an effort profile $y^{2} \in R_{s}^{I}\left(x^{2}\right)$ and an island $L \subseteq V$ in $y^{2}$. By Proposition 4.3 there exists $y^{3} \in R_{s}^{L}\left(y^{2}\right)$ such that, for every $j \in L, y_{j}^{3}=b_{j}\left(y^{3}\right)$. Since $L \cap N_{0}=\emptyset$, at least one of the players in $L$ makes a positive contribution at $y^{3}$, so there is a faction $F^{3} \subseteq L$ in $y^{3}$. Proposition 4.6 implies that, for every $i \in \bar{N}_{F^{3}}, y_{i}^{3}=x_{i}^{1}$, so $F^{3}$ is a faction in $x^{1}$, leading to a contradiction since $F^{3}$ is a proper subset of $F$. Consequently, we have that $s_{0}\left(x^{1}\right)<x_{0}^{1}$.

By Proposition 4.3 there exists $x^{2} \in R_{s}^{I}\left(x^{1}\right)$ such that $x_{0}^{2}=s_{0}\left(x^{1}\right)$ and $x^{2} \in X^{*}$. If no faction in $x^{2}$ has members in both $N_{0}$ and $I \backslash N_{0}$, we are done. Otherwise, there exists $F^{2} \in \mathcal{F}\left(x^{2}\right)$ such that $F^{2} \cap N_{0} \neq \emptyset$ and $F^{2} \backslash N_{0} \neq \emptyset$. By the same argument as in the previous paragraph it must hold that 
$s_{0}\left(x^{2}\right)<x_{0}^{2}$. Since player 0 cannot decrease his efforts forever, we can repeat this argument until we find an effort profile with the desired properties.

Theorem 4.8 (Local impact only). Let $s \in S_{0} \times\left\{b_{I}\right\}$, let $x \in X$ be recurring, and let $y \in[x]_{s}$. For every $i \in I$ such that $\bar{N}_{i} \cap N_{0}=\emptyset$ it holds that $b_{i}(x)=x_{i}=y_{i}=b_{i}(y)$.

Proof. In case $\left|[x]_{s}\right|=1$, the statement holds trivially, so assume $\left|[x]_{s}\right|>1$. By Proposition 4.7 there exists $x^{1} \in[x]_{s} \cap X^{*}$ such that every faction in $x^{1}$ is either completely inside or completely outside $N_{0}$. By Proposition 4.6, for every $F \in \mathcal{F}\left(x^{1}\right)$ such that $F \cap N_{0}=\emptyset$, for every $i \in \bar{N}_{F}$, it holds that $b_{i}(x)=x_{i}=y_{i}=b_{i}(y)$.

Suppose the theorem is false. Then there is a player $j \in I$ such that $\bar{N}_{j} \cap N_{0}=\emptyset$ and $x_{j}^{1}=0$ and there is $x^{2} \in[x]_{s}$ such that $x_{j}^{2}>0$. It follows that there must exist an effort profile $x^{3} \in[x]_{s}$ such that $x_{j}^{3}=0$ and $x^{3}\left(N_{j}\right)<1$, since otherwise the profile $x^{2}$ with $x_{j}^{2}>0$ cannot be reached from $x^{1}$. By Proposition 4.3 there exists $x^{4} \in R_{s}^{I \backslash \bar{N}_{j}}\left(x^{3}\right)$ such that, for every $i \in I \backslash \bar{N}_{j}, x_{i}^{4}=b_{i}\left(x^{4}\right)$. We distinguish two cases.

Case 1: $\min _{i \in N_{j}^{+}\left(x^{4}\right)} c_{i}\left(x^{4}\right)>c_{j}\left(x^{4}\right)=x^{4}\left(N_{j}\right)$. By applying Proposition 4.4 for $J=N_{j}^{+}\left(x^{4}\right)$, we reach an effort profile $x^{5}$ for which $x^{5}\left(N_{j}^{+}\left(x^{4}\right)\right)=x^{5}\left(N_{j}\right)=0$. The profile $x^{6}=\beta_{j}\left(x^{5}\right)$ belongs to $[x]_{s}$ and has $\{j\}$ as a one-player faction, where $\{j\} \cap N_{0}=\emptyset$. By Proposition 4.6 it follows that $x_{j}^{1}=x_{j}^{6}=1$, a contradiction with $x_{j}^{1}=0$.

Case 2: $\min _{i \in N_{j}^{+}\left(x^{4}\right)} c_{i}\left(x^{4}\right) \leq c_{j}\left(x^{4}\right)=x^{4}\left(N_{j}\right)$. For every $i \in N_{\bar{N}_{j}}^{+}\left(x^{4}\right)$ it holds that $x_{i}^{4}=b_{i}\left(x^{4}\right)$, so $c_{i}\left(x^{4}\right)=1$. Since $x^{4}\left(N_{j}\right)=x^{3}\left(N_{j}\right)<1$, there is a valley $V$ in $x^{4}$ with $V \subseteq \bar{N}_{j}$. From $\bar{N}_{j} \cap N_{0}=\emptyset$, it follows that $V \cap N_{0}=\emptyset$. By Lemma A.5 there exists $L \subseteq V$ and there exists $x^{5} \in R_{s}^{I}\left(x^{4}\right)$ such that $L$ is an island at $x^{5}$. By Proposition 4.3 there exists $x^{6} \in R_{s}^{L}\left(x^{5}\right)$ such that, for every $i \in L, x_{i}^{6}=b_{i}\left(x^{6}\right)$. Since $L \cap N_{0}=\emptyset$, at least one of the players in $L$ makes a positive contribution at $x^{6}$, so there is a faction $F^{6} \subseteq L$ in $x^{6}$. Since $F^{6} \cap N_{0}=\emptyset$, it follows by Proposition 4.6 that for every $i \in F^{6}, c_{i}\left(x^{4}\right)=1$. At the same time, $i \in V$, so $c_{i}\left(x^{4}\right) \leq c_{j}\left(x^{4}\right)<1$.

Proposition 5.2. Let $\Gamma^{\prime}(G)$ be such that $G \in \mathcal{G}_{0}$. There exists $\bar{\delta}<1$ such that, for every $\delta>\bar{\delta}$,

$$
\underline{u}(G)=\bar{u}(G)=f_{0}(\bar{e}(G)) .
$$

Proof. For $x \in X$, let $I^{1}(x)=\left\{i \in I: x_{i}=1\right\}$ denote the set of myopic players with effort level 1. Let $M \in \mathcal{K}_{0}(G)$ be an independent set of $N_{0}(G)$ with cardinality $\bar{e}(G)$ and consider the strategy $s_{0} \in S_{0}$ defined by

$$
s_{0}(x)= \begin{cases}0 & \text { if } I^{1}(x) \subseteq M \\ 1 & \text { otherwise }\end{cases}
$$

To show the statement, we first argue that under the strategy profile $s=\left(s_{0}, b_{I}\right)$ the game reaches an effort profile where the farsighted player's instantaneous payoff equals $f_{0}(\bar{e}(G))$ with probability 1. Let $x^{M} \in X$ be the effort profile for which $x_{i}=1$ if $i \in M$ and $x_{i}=0$ otherwise. Notice that 
$A\left(s_{0}\right)=\left\{x^{M}\right\}$ as in every other effort profile the farsighted player or a member of $M$ would change his effort. Furthermore, for every $x \in X$ it holds that $x^{M} \in R_{s}(x)$. In fact, the following sequence of updates shows that it is reachable by fewer than $n+2+\bar{e}(G)$ revisions. Assume $I^{1}(x) \nsubseteq M$. An update by player 0 results in effort level $s_{0}(x)=1$. Next revisions by each of the myopic players in any given order result in them choosing an effort level equal to 0 . This takes $n$ steps and results in an effort profile $x^{\prime}$. Next, player 0 decreases his efforts to $s_{0}\left(x^{\prime}\right)=0$. Finally, the members of $M$ revise in any order to reach the profile $x^{M}$ in $\bar{e}(G)$ more steps. Assume $I^{1}(x) \subseteq M$. Then the members of $M \backslash I^{1}(x)$ revise in any order and reach the profile $x^{M}$ in at most $\bar{e}(G)$ steps. The effort profile $x^{M}$ is absorbing for $s_{0}$. It follows that, for every $\omega \in \Omega$,

$$
\lim _{t \rightarrow \infty} u_{0}^{t}(\omega, s)=f_{0}(\bar{e}(G))
$$

Since there exists a strategy that guarantees a long-run payoff of $f_{0}(\bar{e}(G))$ there is $\bar{\delta}<1$ such that, for every $\delta>\bar{\delta}$, for every $\omega \in \Omega$, for every $\operatorname{SSPE} s^{*} \in S^{*}$,

$$
\lim _{t \rightarrow \infty} u_{0}^{t}\left(\omega, s^{*}\right) \geq \lim _{t \rightarrow \infty} u_{0}^{t}(\omega, s)=f_{0}(\bar{e}(G)) .
$$

On the other hand, $f_{0}(\bar{e}(G))=\max _{x \in X^{\prime}} \pi_{0}(x)$, so by Proposition 3.5 and Remark 3.6 we also have, for every $\omega \in \Omega$, for every $\operatorname{SSPE} s^{*} \in S^{*}$,

$$
\lim _{t \rightarrow \infty} u_{0}^{t}\left(\omega, s^{*}\right) \leq f_{0}(\bar{e}(G))
$$

We conclude that there exists $\bar{\delta}<1$ such that, for every $\delta>\bar{\delta}, \underline{u}(G)=\bar{u}(G)=f_{0}(\bar{e}(G))$.

Proposition 5.6. There exists $\bar{\delta}<1$ such that, for every $\delta>\bar{\delta}$, the lower bound on the farsighted player's expected payoffs in the game $\Gamma^{\prime}(G)$ is given by

$$
\underline{u}(G)= \begin{cases}f_{0}(\underline{e}(G)) & \text { if } \underline{e}(G)>0, \\ f_{0}(1)-\gamma_{0} & \text { if } \underline{e}(G)=0 .\end{cases}
$$

Proof. We first consider the case where $\underline{e}(G)=0$. In any network the farsighted player can guarantee the long-run payoff $f_{0}(1)-\gamma_{0}$ by making an effort of $s_{0}(x)=1$ for every $x \in X$. Hence, there exists $\bar{\delta}<1$ such that, for every $\delta>\bar{\delta}$, we have $\underline{u}(G) \geq f_{0}(1)-\gamma_{0}$. Let $x^{\mathrm{b}} \in X^{\mathrm{b}}$ be such that $\bar{e}\left(\left.G\right|_{I_{0}^{\mathrm{u}}\left(x^{\mathrm{b}}\right)}\right)=0$, which happens if and only if $N_{0}(G) \cap I_{0}^{\mathrm{u}}\left(x^{\mathrm{b}}\right)=\emptyset$. When the action profile is equal to $x^{\mathrm{b}}$, player 0 acts as if he was the only player as he gets a contribution of 0 from his neighbors and no player will change his effort level no matter what player 0 does. Hence, in every SSPE $s^{*} \in S^{*}$ we must have $s_{0}^{*}\left(x^{\mathrm{b}}\right)=1$, meaning that $\underline{u}(G) \leq f_{0}(1)-\gamma_{0}$ also holds.

Now consider the case $\underline{e}(G)>0$. We show first that $\underline{u}(G) \leq f_{0}(\underline{e}(G))$. Let $x^{\mathrm{b}} \in X^{\mathrm{b}}$ be such that $\underline{e}(G)=\bar{e}\left(\left.G\right|_{I_{0}^{\mathrm{u}}\left(x^{\mathrm{b}}\right)}\right)$. At effort profile $x^{\mathrm{b}}$, in every SSPE the players outside $I_{0}^{\mathrm{u}}\left(x^{\mathrm{b}}\right)$ never change their efforts and no player in $I_{0}^{\mathrm{u}}\left(x^{\mathrm{b}}\right)$ is connected to a player with positive efforts outside $I_{0}^{\mathrm{u}}\left(x^{\mathrm{b}}\right)$. Therefore, the players inside $I_{0}^{\mathrm{u}}\left(x^{\mathrm{b}}\right)$ do not affect the players outside $I_{0}^{\mathrm{u}}\left(x^{\mathrm{b}}\right)$ in any way. We can therefore remove 
the players outside $I_{0}^{\mathrm{u}}\left(x^{\mathrm{b}}\right)$ from the network and study the network $\left.G\right|_{I_{0}^{\mathrm{u}}\left(x^{\mathrm{b}}\right)}$. Since $I_{0}^{\mathrm{u}}\left(x^{\mathrm{b}}\right) \subseteq \bar{N}_{0}(G)$, we can apply Proposition 5.2. Hence, there exists $\bar{\delta}<1$ such that, for every $\delta>\bar{\delta}$,

$$
\underline{u}\left(\left.G\right|_{I_{0}^{\mathrm{u}}\left(x^{\mathrm{b}}\right)}\right)=f_{0}\left(\bar{e}\left(\left.G\right|_{I_{0}^{\mathrm{u}}\left(x^{\mathrm{b}}\right)}\right)\right) .
$$

We find that

$$
\underline{u}(G) \leq \underline{u}\left(\left.G\right|_{I_{0}^{\mathrm{u}}\left(x^{\mathrm{b}}\right)}\right)=f_{0}(\underline{e}(G)) .
$$

We show next that there exists a strategy for the farsighted player which achieves long-run payoffs greater than or equal to $\underline{u}(G)$, irrespective of the initial state. From this it follows that there exists $\bar{\delta}<1$ such that, for every $\delta>\bar{\delta}, \underline{u}(G) \geq f_{0}(\underline{e}(G))$, which completes the proof.

For $n=1$, the strategy $s_{0} \in S_{0}$ such that $s_{0}(x)=0$ for every $x \in X$ achieves long-run payoffs greater than or equal to $\underline{u}(G)=f_{0}(\underline{e}(G))=f_{0}(1)$.

Now fix $n$ and assume that for every network $G$ with fewer than $n$ players there is a strategy which achieves long-run payoffs greater than or equal to $f_{0}(\underline{e}(G))$, irrespective of the initial state.

Let $G$ be a network with $n$ myopic players that belongs to $\mathcal{G}_{0}$. Let $M \in \mathcal{K}_{0}(G)$ be an independent set of $N_{0}(G)$ with cardinality $\underline{e}(G)=\bar{e}(G)$ and consider the strategy $s_{0} \in S_{0}$ defined by

$$
s_{0}(x)= \begin{cases}0 & \text { if } I^{1}(x) \subseteq M, \\ 1 & \text { otherwise }\end{cases}
$$

It is shown in the proof of Proposition 5.2 that $s_{0}$ achieves long-run payoffs equal to $f_{0}(\underline{e}(G))$ irrespective of the initial state.

Now let $G$ be a network with $n$ myopic players that does not belong to $\mathcal{G}_{0}$ and let some state $\left(x^{0}, j\right) \in \Omega$ be given. Let $x^{1}=\beta_{j}\left(x^{0}\right)$. If $\bar{B}\left(x^{1}\right) \neq \emptyset$, then, by the induction hypothesis, the farsighted player has a strategy for the network $\left.G\right|_{I_{0} \backslash \bar{N}_{\bar{B}\left(x^{1}\right)}(G)}$ which achieves long-run payoffs greater than or equal to $\underline{u}\left(\left.G\right|_{I_{0} \backslash \bar{N}_{\bar{B}\left(x^{1}\right)}(G)}\right)=f_{0}\left(\underline{e}\left(\left.G\right|_{I_{0} \backslash \bar{N}_{\bar{B}\left(x^{1}\right)}(G)}\right)\right)$. Since effort levels of players in $\bar{B}\left(x^{1}\right)$ are unaffected, this strategy can be applied to the network $G$ in a straightforward way and achieves long-run payoffs greater than or equal to

$$
\underline{u}\left(\left.G\right|_{I_{0} \backslash \bar{N}_{\bar{B}\left(x^{1}\right)}(G)}\right)=f_{0}\left(\underline{e}\left(\left.G\right|_{I_{0} \backslash \bar{N}_{\bar{B}\left(x^{1}\right)}(G)}\right)\right) \geq f_{0}\left(\min _{x \in X^{\mathrm{b}}} \bar{e}\left(\left.G\right|_{I_{0}^{\mathrm{u}}(x)}\right)\right)=f_{0}(\underline{e}(G))
$$

where the inequality follows from the fact that any blocking profile in $\left.G\right|_{I_{0} \backslash \bar{N}_{\bar{B}\left(x^{1}\right)}(G)}$ can be extended to a blocking profile in $G$ in the straightforward way.

Consider the case $\bar{B}\left(x^{1}\right)=\emptyset$. Let the farsighted player use a strategy $s_{0} \in S_{0}$ which is such that, for every $x \in X$ with $\bar{B}(x)=\emptyset, s_{0}(x)=1$ if $x\left(N_{0}(G)\right)>0$ and $s_{0}(x)=0$ if $x\left(N_{0}(G)\right)=0$. Let $s=\left(s_{0}, b_{I}\right)$. We show that under $s$ an effort profile with a non-empty closed partnership can be reached from $x^{1}$ after which the proof follows along the same lines as in the previous paragraph.

We first show that there is $y \in R_{s}\left(x^{1}\right)$ with $y_{0}=0$ and $y\left(N_{0}(G)\right)=0$. If $x^{1}\left(N_{0}(G)\right)=0$, then let player 0 update and we are done. If $x^{1}\left(N_{0}(G)\right)>0$, then let player 0 update first, leading to a profile 
$x^{2}$ with $x_{0}^{2}=1$. Next let all players $i \in N_{0}(G)$ with $x_{i}^{2}>0$ update sequentially, ultimately leading to a profile $x^{3}$ with $x^{3}\left(N_{0}(G)\right)=0$. Then let player 0 update to generate the desired effort profile.

Let $y \in X$ be an effort profile with $y_{0}=0$ and $y\left(N_{0}(G)\right)=0$. If, for every $i \in I, y_{i}=0$, then choose a player $j^{1} \in I \backslash N_{0}(G)$ to update. For the resulting effort profile $y^{1}=\beta_{j^{1}}(y)$ it holds that $\bar{B}\left(y^{1}\right)=\left\{j^{1}\right\}$, so a closed partnership has been reached.

If there are myopic players with positive contributions, then let all these players sequentially update until they all play best responses at a profile $y^{2}$, see Proposition 4.3 . There is at least one player with a positive contribution at $y^{2}$, so $B\left(y^{2}\right) \neq \emptyset$. If $\bar{B}\left(y^{2}\right) \neq \emptyset$, then we are done. Otherwise, let $j^{2}$ be a neighbor of $B\left(y^{2}\right)$ with $c_{j^{2}}\left(y^{2}\right)<1$. Let $j^{2}$ absorb the set of players $J=N_{j^{2}}(G) \cap B\left(y^{2}\right)$ as in Proposition 4.4 and denote the resulting effort profile by $y^{3}$, so $y_{j^{2}}^{3}=1$ and $y^{3}\left(N_{j^{2}}(G)\right)=0$. If $j^{2} \in I \backslash N_{0}(G)$, then $\bar{B}\left(y^{3}\right)=\left\{j^{2}\right\}$, so we are done. If $j^{2} \in N_{0}(G)$, then notice first that $c_{j^{2}}\left(y^{2}\right)<1$ implies $B\left(y^{2}\right) \backslash J \neq \emptyset$. Let 0 be the next updating player, followed by $j^{2}$, followed by 0 , reaching a profile $y^{4}$ with $y_{0}^{4}=y_{j^{2}}^{4}=0$. Next let all myopic players with positive contributions at $y^{4}$ sequentially update until they all play best responses at a profile $y^{5}$, see Proposition 4.3 . We have that $\emptyset \neq B\left(y^{5}\right) \subsetneq B\left(y^{2}\right)$. Now repeating this argument a finite number of times will eventually result in an effort profile with a non-empty closed partnership.

\section{References}

Allouch, N., 2015. On the private provision of public goods on networks. Journal of Economic Theory, $157,527-552$.

Allouch, N., and King, M., 2019. A network approach to welfare. BSG Working Paper Series, BSGWP-2019/027.

Ballester, C., Calvó-Armengol, A., and Zenou, Y., 2006. Who's who in networks. Wanted: The key player. Econometrica, 74, 1403-1417.

Bayer, P., Herings, P.J.J., Peeters, R. and Thuijsman, F., 2019. Adaptive Learning in Weighted Network Games. Journal of Economic Dynamics and Control, 105, 250-264.

Bervoets, S., Bravo, M., and Faure, M., 2016. Learning and convergence to Nash in games with continuous action sets. Working paper.

Bervoets, S., and Faure, M., 2019. Stability in games with continua of equilibria. Journal of Economic Theory, 179, 131-162..

Bloch, F., and van den Nouweland, A., 2019. Farsighted stability with heterogeneous expectations. Working paper. 
Blume, L.E., Brock, W.A., Durlauf, S.N., and Ioannides, Y.M., 2010. Identification of social interactions. In Benhabib, J., Bisin, A., and Jackson, M.O. (eds) Handbook of Social Economics. Amsterdam: North Holland. pp. 853-964.

Bramoullé, Y., and Kranton, R., 2007. Public goods in networks. Journal of Economic Theory, 135, 478-494.

Bramoullé, Y., Kranton, R., and D'Amours, M., 2014. Strategic interaction and networks. American Economic Review, 104, 898-930.

Camerer, C., Ho, T.H., and Chong, J.K., 2002. Sophisticated experience-weighted attraction learning and strategic teaching in repeated games. Journal of Economic Theory, 104, 137-188.

Calvó-Armengol, A., and Zenou, Y., 2004. Social networks and crime decisions: The role of social structure in facilitating delinquent behavior. International Economic Review, 45, 939-958.

Choi, S., Goyal, S., and Moisan, F., 2019. Network Formation in Large Groups. Cambridge Working Papers in Economics CWPE1935, 1-76.

Dutta, B., Ghosal, S., and Ray, D., 2005. Farsighted network formation. Journal of Economic Theory, 122, 143-164.

Dutta, B., and Vohra, R., 2017. Rational expectations and farsighted stability. Theoretical Economics, $12,1191-1227$.

Ellison, G., 1997. Learning from personal experience: One rational guy and the justification of myopia. Games and Economic Behavior, 19, 180-210.

Eshel, I., Samuelson, L., and Shaked, A., 1998. Altruists, egoists, and hooligans in a local interaction model. American Economic Review, 88, 157-179.

Fudenberg, D., and Levine, D., 1998. The Theory of Learning in Games, MIT Press, Cambridge.

Galeotti, A., and Goyal, S., 2010. The law of the few. American Economic Review, 100, 1468-1492.

Goyal, S., 2012. Connections: An Introduction to the Economics of Networks. Princeton University Press.

Herings, P.J.J., Mauleon, A., and Vannetelbosch, V., 2009. Farsightedly stable networks. Games and Economic Behavior, 67, 526-541.

Herings, P.J.J., Mauleon, A., and Vannetelbosch, V., 2017. Matching with myopic and farsighted players. Working paper.

Hofstadter, D., 1983. Dilemmas for superrational thinkers, leading up to a luring lottery. Scientific American, 248 - reprinted in: Hofstadter, D., 1985. Metamagical Themas. Basic Books. 737-755. 
Jackson, M.O., and Zenou, Y., 2014. Games on networks. In Young, P., and Zamir, S. (eds) Handbook of Game Theory, Vol. 4, Elsevier Science. pp. 91-157.

Klaus, B., Klijn, F., and Walzl, M., 2011. Farsighted stability for roommate markets. Journal of Public Economic Theory, 13, 921-933.

König, M.D., Liu, X., and Zenou, Y., 2019. R\&D networks: Theory, empirics and policy implications. Review of Economics and Statistics, 101, 476-491.

Luo, C., Mauleon, A., and Vannetelbosch, V., 2018. Network formation with myopic and farsighted players. Working paper.

Mauleon, A., and Vannetelbosch, V., 2004. Farsightedness and cautiousness in coalition formation games with positive spillovers. Theory and Decision, 56, 291-324.

Page Jr, F.H., Wooders, M.H., and Kamat, S., 2005. Networks and farsighted stability. Journal of Economic Theory, 120, 257-269.

Parise, F., and Özdaglar, A., 2019. A variational inequality framework for network games: Existence, uniqueness, convergence and sensitivity analysis. Games and Economic Behavior, 114, 47-82.

Puterman, M.L., 1994. Markov Decision Processes, Discrete Stochastic Dynamic Programming. John Wiley and Sons, Hoboken, New Jersey.

Ray, D., and Vohra, R., 2015. The farsighted stable set. Econometrica, 83, 977-1011.

Sandler, T., and Hartley, K., 1995. The Economics of Defense. Cambridge University Press.

Sandler, T., and Hartley, K., 2001. Economics of alliances: The lessons for collective action. Journal of Economic Literature, 39, 869-896.

Sandler, T., and Hartley, K., 2007. Handbook of Defense Economics: Defense in a Globalized World. Elsevier.

Voorneveld, M., 2000. Best-response potential games. Economic Letters, 66, 289-295. 